\title{
Detecting Amino Acid Preference Shifts with Codon-Level Mixture Models
}

\author{
by \\ Shan Omar Kazmi
}

A thesis submitted to the Faculty of Graduate and Postdoctoral Affairs in partial

fulfillment of the requirements for the degree of

\author{
Master of Science \\ in
}

Biology with Specialization in Bioinformatics

\author{
Carleton University \\ Ottawa, Ontario \\ (C)2017 Shan Omar Kazmi
}




\begin{abstract}
In recent years, increasing attention has been placed on the development of phylogenybased statistical methodologies for uncovering site-specific changes in amino acid fitness profiles over time. The few available random-effects approaches, modelling across-site variation in amino acid profiles as random variables drawn from a statistical law, either lack a mechanistic codon-level formulation, or pose significant computational challenges. Here, we explore a simple and fast method based on a predefined finite mixture of amino acid profiles within a codon-level mutation-selection substitution model. Our study detects shifts of amino acid profiles over a known subclade of a tree, using simulations with and without shifts over the sub-clade to study the properties of the method. We apply the approach to a real data set, previously studied with other methods: lists of sites identified as having undergone a change in amino acid profile have obvious overlap between methods, while also showing notable differences.
\end{abstract}




\section{Contents}

Title Page

\begin{tabular}{lll}
\hline Abstract ii & ii
\end{tabular}

$\begin{array}{lll}1 & \text { Introduction } & 1\end{array}$

1.1 Markov Substitution Processes . . . . . . . . . . . . . . . . . . . . . 2

$1.2 \quad$ Bayesian Inference and Markov Chain Monte Carlo . . . . . . . . . . 5

1.2.1 Bayes' Theorem . . . . . . . . . . . . . . . . 5

1.2.2 Marginalization of Nuisance Parameters . . . . . . . . . 5

1.2 .3 Markov Chain Monte Carlo . . . . . . . . . . . . . . 6

1.3 Advanced Models . . . . . . . . . . . . . . . . . . . . . . . 7

1.3 .1 Mixture Models . . . . . . . . . . . . . . . . . . 7

1.3 .2 Empirical Mixture Models . . . . . . . . . . . . . . . . . 8

1.3.3 Mutation-Selection Codon Models . . . . . . . . . . . . . . . . 9

1.3 .4 Preference Shifts and Time-Heterogeneity . . . . . . . . . . . 12

2 Detecting Amino Acid Preference Shifts with Codon-Level Mixture

$\begin{array}{ll}\text { Models } & 14\end{array}$

2.1 Introduction . . . . . . . . . . . . . . . . . . . . . . . 14

2.2 Materials and Methods . . . . . . . . . . . . . . . . . . . . 17

2.2 .1 Data . . . . . . . . . . . . . . . . . 17 
2.2 .2 Model . . . . . . . . . . . . . . . . . . . . . . . 18

$2.2 .3 \quad$ Probability of Identical Profiles $\ldots \ldots \ldots \ldots$

2.2 .4 Simulations . . . . . . . . . . . . . . . . . . 21

2.3 Results and Discussion . . . . . . . . . . . . . . . . . . . . . . 21

2.3 .1 Simulation Data . . . . . . . . . . . . . . . . . . . . . 21

2.3 .2 Real Data . . . . . . . . . . . . . . . . . . . . . . 30

2.4 Conclusion $\ldots \ldots \ldots \ldots \ldots \ldots$

\begin{tabular}{lll}
\hline 3 & Future Directions & 37
\end{tabular}

$3.1 \quad$ Empirical Profiles in a Mutation-Selection Context $\ldots . . . . . .37$

3.2 Nonstationary Substitution Models . . . . . . . . . . . . . . . . 38

3.3 Substitution Mapping. . . . . . . . . . . . . . . . . . . . . . . . . . . . . . 39

3.3 .1 Preliminary Results . . . . . . . . . . . . . . . . . . . 40 


\section{List of Figures}

2.1 Influenza PB2 phylogenetic tree, with monophyletic human-host clade. Taken from Tamuri et al.[(2009)] . . . . . . . . . . . . . . . . . . 18

2.2 Logoplots of MutSelBC profile sets with four different "peakedness" values, indicating probability mass of the chosen amino acids in each profile. For each profile set, the probability of the profile-specific amino acid is $50 \%, 75 \%, 90 \%$ and $99.9 \%$ respectively. . . . . . . . . . . . . . 23

2.3 Allocation probability logoplots for the same codon site using MutSelBC profiles with 90\% (top), 75\% (middle) and 50\% (bottom) "peakedness" values. For each logoplot, the solid bar indicates the allocation probability to a given profile at that site, and the letters underneath indicate the profile. The letters are scaled to indicate the probability of that amino acid at the site in that profile. . . . . . . . . . . . . . . 24

2.4 (a) True positive, (b) true negative, (c) false positive and (d) false negative detection of preference shifts, by peakedness value, for simulated data using MutSelBC profiles. . . . . . . . . . . . . . . . . . 27

2.5 (a) True positive, and (b) false positive detection of preference shifts value, for simulated data using C10, C20, C40 and C60 profiles. . . . 28 
$2.6 \quad$ Receiver operating characteristic plot for PIP thresholds used to define a preference shift. Data was simulated with peakedness values ranging from 0.50 to 0.999 . A PIP threshold of 0.05 was determined to acceptably encompass preference shifts in all peakedness values. . . . 29

2.7 Allocation probability logoplot using MutSelC60 (top) and MutSelBC (bottom) profiles for codon site 661. Red is avian-host strains and blue is human-host strains of Influenza PB2. Purple indicates overlapping profile allocation in both clades. . . . . . . . . . . . . . . . . . . . . . 31

2.8 Allocation probability logoplots in avian (red) and human (blue) strains of Influenza PB2. Purple indicates overlapping profile allocation in both clades. At top is a strongly identified preference shift with a PIP of 0.865. At middle is a strongly identified non-shift, with a PIP of 0.00. At bottom, the low PIP of 0.115 does not indicate a preference shift, as site is weakly allocated to a number of profiles in both clades.

3.1 (a) True positive, (b) true negative, (c) false positive and (d) false negative detection of preference shifts for simulated data using substitution mapping. . . . . . . . . . . . . . . . . . . . . . . . 41 


\section{List of Tables}

2.1 Codon sites with preference shifts detected with MutSelC60 and MutSelBC profiles, and sites identified in previous studies (Finkelstein et al.| $(2007)$ and Tamuri et al.[(2009)). . . . . . . . . . . . . . 33 


\section{Chapter 1}

\section{Introduction}

Modern advances in gene sequencing technology have allowed rapid acquisition of extensive amounts of genetic data. This data, stored in large-scale genomic collections such as the National Center for Biotechnology Information (NCBI, 2017), provide the means to quantitatively investigate underlying biological structures and processes in detail. Coupled with ever-increasing advances in computational hardware and algorithm efficiency, we are able to ask, and answer, questions about biology to an unprecedented level of detail.

One major approach in this research is the use of multiple sequence alignments in comparison and inference of evolutionary processes. In these alignments, nucleotide or amino acid sequences from related organisms are organized such that each column in an array corresponds to a specific position from a particular species. Each of these sequences is not considered to be independent from another; rather, they share a complex structure of dependency represented as a genealogy between these organisms, referred to as a phylogenetic tree. We can use this tree to describe a mathematical process that runs along the branches of the phylogeny, which characterizes the timing and nature of the evolutionary events by which the sequences are related. The sequence alignment itself, for analytical purposes, can therefore be considered a 
realization of this mathematical process.

\subsection{Markov Substitution Processes}

The evolutionary processes that produce genetic sequences, such as the waiting time between substitution events, and the nature of the substitution events along the branches of the phylogenetic tree, can be represented as a continuous-time first-order Markov process. In a first-order Markov process, change in a system from one state to the next is dependent on the current state, but not on any previous state, i.e. it has no "memory". In a gene sequence, which has a state space over the four possible nucleotides, the Markov process consists of a stationary probability vector, i.e. the amount of time the process spends in each state (which we denote here as $\varphi=\left(\varphi_{i}\right)_{1 \leq i \leq 4}$, with the constraint $\left.\sum_{1 \leq i \leq 4} \varphi_{i}=1\right)$, and a transition rate matrix $Q$, which describes the instantaneous rate of change from state $i$ to $j\left(q_{i j}\right)$. The Markov processes we work with here are time-reversible, which implies that $\varphi_{i} q_{i j}=\varphi_{j} q_{j i}$, and therefore that the likelihood function (explained below) is invariant to the orientation of the Markov process over the tree.

Several implementations of nucleotide substitution Markov models have been devised. The simplest, the JC69 model (Jukes et al., 1969), assumes that every nucleotide changes to every other nucleotide at the same rate, giving a rate matrix of

$$
Q=q_{i j}=\left[\begin{array}{cccc}
- & \lambda & \lambda & \lambda \\
\lambda & - & \lambda & \lambda \\
\lambda & \lambda & - & \lambda \\
\lambda & \lambda & \lambda & -
\end{array}\right],
$$

where $\lambda$ is a factor that ensures that branch lengths of the phylogeny represent the expected number of substitutions. The diagonals in the rate matrix are the negative sum of off-diagonal entries in each respective row, which ensures that each row sums 
to zero.

The F81 model (Felsenstein, 1981) uses the base frequency of each nucleotide, that is, the probability of substitution to that nucleotide in the sequence, to determine the rate of change to that nucleotide. These base frequencies vary independently, giving a rate matrix of

$$
Q=q_{i j}=\left[\begin{array}{cccc}
- & \varphi_{C} & \varphi_{G} & \varphi_{T} \\
\varphi_{A} & - & \varphi_{G} & \varphi_{T} \\
\varphi_{A} & \varphi_{C} & - & \varphi_{T} \\
\varphi_{A} & \varphi_{C} & \varphi_{G} & -
\end{array}\right]
$$

where $\varphi$ is the base or equilibrium frequency of the nucleotide in question.

It is well known that the rate of substitutions varies between transitions, where two purine or pyrimidine nucleotides exchange, and transversions, where a purine is substituted for a pyrimidine, or vice versa (Kimura, 1980). Hasegawa et al. (1985) combined a single parameter $\kappa$, representing the ratio of transitions to transversions, with F81 to create a model (HKY85) where substitutions are specified by both the transition/transversion ratio and frequency:

$$
Q=q_{i j}=\left[\begin{array}{cccc}
- & \varphi_{C} & \kappa \varphi_{G} & \varphi_{T} \\
\varphi_{A} & - & \varphi_{G} & \kappa \varphi_{T} \\
\kappa \varphi_{A} & \varphi_{C} & - & \varphi_{T} \\
\varphi_{A} & \kappa \varphi_{C} & \varphi_{G} & -
\end{array}\right]
$$

The General Time-Reversible model (Tavaré, 1986) unifies these into a model with a vector of equilibrium base frequencies and a separate parameter for each pair of nucleotides' exchangeability rates, represented here as $\left(\rho_{i j}\right)_{1 \leq i, j \leq 4}$ with the constraint 
that $\sum_{1 \leq i<j \leq 4} \rho_{i j}=1$ :

$$
Q=q_{i j}=\left[\begin{array}{cccc}
- & \rho_{i j} \varphi_{C} & \rho_{i j} \varphi_{G} & \rho_{i j} \varphi_{T} \\
\rho_{i j} \varphi_{A} & - & \rho_{i j} \varphi_{G} & \rho_{i j} \varphi_{T} \\
\rho_{i j} \varphi_{A} & \rho_{i j} \varphi_{C} & - & \rho_{i j} \varphi_{T} \\
\rho_{i j} \varphi_{A} & \rho_{i j} \varphi_{C} & \rho_{i j} \varphi_{G} & -
\end{array}\right]
$$

These models assume that the substitution processes are strictly nucleotide-level, and ignore the potential coding nature of the DNA sequences, such as in proteincoding genes. Other models work with the translated amino acid sequences, often invoking the same form of the models above, with a 20x20 vector of stationary probabilities, and with the exchangeability parameter $\rho$ having a dimension of 190 .

Once the rate matrix $Q$ has been defined, the probability of the data (that is, the multiple sequence alignment), conditional on the parameters in $Q$ and the topology $(\tau)$ and branch lengths $(\lambda)$ of the phylogenetic tree, can be computed using Felsenstein (1981)'s pruning algorithm. This is known as the likelihood function.

For a given position, matrix exponentiation methods are used to compute transition probabilities over certain branch lengths. Given an internal node state configuration, transition probabilities are multiplied across all branches, and a dynamic programming scheme is employed to sum the resulting product over all internal node states. This provides the probability of a particular data column $p\left(D_{n} \mid \theta\right)$, which then leads to the overall likelihood function, as a product across columns, $p(D \mid \theta)=\prod_{n=1}^{N} p\left(D_{n} \mid \theta\right)$, under the assumption of independence. 


\subsection{Bayesian Inference and Markov Chain Monte Carlo}

\subsubsection{Bayes' Theorem}

Parameters of substitution models can be estimated from sequence alignment data by maximum likelihood statistical methods or by use of Bayesian inference. We will focus here on Bayesian inference, as this is the framework used for our research in the next chapter.

Bayes' theorem states that if given a set of observed data $D$, the probability of a set of parameters $\theta$, known as the posterior probability $p(\theta \mid D)$, is computed from the prior probability $p(\theta)$ and the likelihood function:

$$
p(\theta \mid D)=\frac{p(D \mid \theta) p(\theta)}{\int_{\Theta} p(D \mid \theta) p(\theta) d \theta}
$$

This describes a system known as Bayesian inference, which rests on the view that probability represents states of knowledge about certain parameters of a model. Prior probabilities correspond to our state of knowledge about parameter values before having observed any data, while posterior probabilities correspond to our state of knowledge after acquiring observational data.

The denominator in Bayes' theorem is a high-dimensional integral that is intractable. In practice, therefore, computing the posterior distribution is accomplished using numerical methods, such as Monte Carlo methods (described below).

\subsubsection{Marginalization of Nuisance Parameters}

One major advantage of using Bayesian inference is that we can derive probabilities of parameters of a model that are conditional on other parameters or processes, without 
having to calculate those probabilities directly, by integrating them out as part of the marginal probability (see, e.g. Yang, 2014). Typically in phylogenetics, one is interested in the probability of a particular tree topology $\tau$. On the basis of our joint posterior distribution, we can calculate this probability by integrating away all other parameters involved in the model. These parameters other than $\tau$ are thus considered to be nuisance parameters, in the sense that they are not of direct interest in this calculation. We express the probability of the topology as:

$$
p(\tau \mid \theta)=\int_{\Theta} p(\tau, \theta \mid D) d \theta .
$$

\subsubsection{Markov Chain Monte Carlo}

A conceptually straightforward Markov chain Monte Carlo method is the Metropolis algorithm (Metropolis et al., 1953):

Given an initial parameter state $\theta$ :

- Propose a new state $\theta^{\prime}$, and calculate the ratio of posterior probabilities $(\alpha=$ $\left.p\left(\theta^{\prime} \mid D\right) / p(\theta \mid D)\right)$.

- If $\alpha>1$ then set the state to $\theta^{\prime}$,

- If $\alpha<1$, set the state to $\theta^{\prime}$ with probability $\alpha$,

- else, set the state to $\theta$.

This produces a Markov chain which will have a stationary distribution that is the same as the posterior distribution of $p(\theta \mid D)$. This algorithm was extended by Hastings (1970) to account for biases in the proposal mechanism: with the Metropolis algorithm, proposal mechanisms are symmetrical, in the sense that proposing $\theta^{\prime}$ given $\theta$ will have the same probability as proposing $\theta$ given $\theta^{\prime}$. When this symmetry does not hold, a factor corresponding to the ratio of proposal probabilities is included, known as the Hastings ratio. 


\subsection{Advanced Models}

We have discussed the basic substitution models and computational framework that make up the foundations of our research. In this section we now overview some more advanced models that form the basis of the mutation-selection model that we use in the following chapter.

\subsubsection{Mixture Models}

The models presented above assume that the substitution process is the same at all sites in a sequence, which is clearly not a realistic approximation. Yang 1993 , 1994, 1996) described multiple models which could accommodate site-heterogeneous substitution rates, where the rate at each site could be a random variable drawn from either a continuous gamma distribution, previously defined discrete classes of rates, or a discrete set of rates approximating a gamma distribution. These random variables appear in the model as multipliers on the branch lengths of the phylogenetic tree. Under a site-homogeneous model, these modifiers are 1, whereas under the gammadistributed rates models, branch lengths will "appear" longer or shorter when the rate is above or below 1 .

These models only address global rates of substitution, regardless of the states involved, and assume a site-homogeneous state frequency distribution. This does not account for the fact that we know that constraints and selective factors act to favour specific amino acids at specific sites (Fitch and Margoliash, 1967). Halpern and Bruno (1998) addressed site-heterogeneity with a model which allowed each individual site in a sequence an independent equilibrium frequency. However, a major drawback to this approach is that inference under this model needs large amounts of data: each site in the sequence alignment is associated with its own vector of amino acid

propensities, which leads to potentially hundreds or thousands of parameters to be 
estimated (Lartillot and Philippe, 2004). Furthermore, addition of new sites to the data requires new parameters to be added to the model, which implies that the model has no asymptotic conditions. (Felsenstein, 2001; Rodrigue, 2013).

Mixture models of amino acid frequency vectors can accommodate the issues in site-specific models such as those of Halpern and Bruno (1998) without requiring a separate set of parameters for each datum. The CAT model (Lartillot and Philippe, 2004 ) is a mixture model in which there are $K$ classes, each one a distinct vector of stationary amino acid probabilities. One can then adjust the richness of the heterogeneity in the substitution model by adjusting the number of available classes. Using a Dirichlet process prior allows $K$ to be a free variable in an Markov Chain Monte Carlo algorithm, allowing the data to "choose" the number of classes, providing the ability to limit the number of parameters. The frequency vectors in this Dirichlet process mixture can potentially model multiple sites at once, creating "profiles" of frequencies, allowing the model to generalize to an arbitrary number of added sites. The CAT model of frequency profiles can be combined with state exchangeability parameters, to produce a model referred to as CAT-GTR (Lartillot and Philippe, 2004, Lartillot et al., 2013).

\subsubsection{Empirical Mixture Models}

Quang et al. (2008) explored a simpler, more intuitive mixture modelling approach, consisting of a fixed, finite set of $K$ components. Their rationale was to obtain a set of values by maximum likelihood, based on a large dataset, deriving an empirical mixture that could then be reused in subsequent analyses. This is in contrast to the CAT model which re-infers a mixture for each new analysis. Quang et al. (2008) ran their algorithm on models with a fixed number of classes ranging from 10 to 60 . They found that the bulk of the statistical improvement in their study was achieved with a model including 20 components, dubbed C20, but noticed continuous but 
less dramatic improvements with models including up to 60 components. This C60 mixture model is used as a basis for our work in the next chapter.

\subsubsection{Mutation-Selection Codon Models}

The models discussed above operate in either the nucleotide state space, or the translated amino acid state space. Codon models use nucleotide data but acknowledge the coding nature of these sequences, by reformulating the nucleotide state space into one based on triplets of nucleotides. Rates from one triplet to another may depend on whether they are synonymous, encoding the same amino acid, or non-synonymous, encoding a different amino acid.

In these models mutations to stop codons are omitted, as they are typically considered lethal, giving a Markov model with state space size of 61 in the universal genetic code. Goldman and Yang (1994) devised a model which omitted the rare case of a two- or three-nucleotide substitution in a single event, focusing on point-mutation events. The commonly used version of this model incorporates two substitution parameters: $\kappa$, controlling transition/transversion ratio, and $\omega$, controlling the ratio between synonymous and nonsynonymous codon substitution, as well as a $\pi$ parameter controlling frequency of the target codon. For a substitution from codon i to codon $\mathrm{j}$, this gives a rate of:

$$
q_{i j}= \begin{cases}0, & \text { if the codons differ by more than one position, } \\ \pi_{j}, & \text { if the difference is a synonymous transversion, } \\ \kappa \pi_{j}, & \text { if a synonymous transition, } \\ \omega \pi_{j}, & \text { if a nonsynonymous transversion, } \\ \omega \kappa \pi_{j}, & \text { if a nonsynonymous transition. }\end{cases}
$$

Codon equilibrium frequencies can be specified in multiple ways. The most common approach treats codon frequency as a simple product of the frequencies of the 
three nucleotides that comprise the codon, written as $\pi_{i} \propto \varphi_{i_{1}} \varphi_{i_{2}} \varphi_{i_{3}}$. This system, called $\mathrm{F} 1 \times 4$, includes a single frequency vector of four nucleotide values to compute all codon frequencies. A slightly more complex system, called F3×4, includes a separate set of frequencies for each nucleotide position in a codon (Yang, 1997).

Muse and Gaut (1994) described a similar model which treated codons explicitly as their component nucleotides, expressing substitutions as a function of the target nucleotide frequency at a given codon position. In this model, the rate of substitution from codon i to codon $\mathrm{j}$ at position $\mathrm{c}$ would be:

$$
q_{i j}= \begin{cases}0 & \text { if the codons differ by more than one position, } \\ \varphi_{j_{c}} & \text { if synonymous, } \\ \omega \varphi_{j_{c}} & \text { if non-synonymous }\end{cases}
$$

where $\varphi_{j_{c}}$ is the frequency of nucleotide $j$ at position $c$, and $\omega$ is the nonsynonymous/synonymous ratio.

These codon models only differentiate between synonymous and nonsynonymous events, but do not address the fact that nonsynonymous events are often replacements between specific amino acids. A recent trend in codon modelling attempts to recognize the nature of the amino acid replacement implied by a mutation. Yang and Nielsen (2008) refer to these as mutation-selection models, involving parameters controlling the mutational process, which recognize the distinct nature of the mutation, and parameters controlling selection at the amino acid level, which recognize the nature of the amino acids involved.

Rodrigue et al. (2010) developed a nonparametric model (designated MG-MutSelDP) for a codon level mutation-selection framework to address the limitations of previous codon models, particularly in their site-homogeneous equilibrium frequencies. These mutation-selection models are focused on modelling purifying selection, in contrast to the $\omega$ based models, which more crudely model purifying selection and adaptive se- 
lection. In recent works, mutation-selection models have been used as a null to detect more subtle adaptive processes, by measuring the departure of non-synonymous rates from those implied by their rendering of purifying selection Bloom, 2017, Rodrigue and Lartillot, 2017).

Rodrigue et al. (2010)'s model consists of pairwise nucleotide exchangeability parameters, and nucleotide propensity parameters. The nucleotide parameters follow the same form as the GTR model. Amino acid frequency parameters are reformulated into fitness profiles following a Dirichlet process analogous to that of Lartillot and Philippe (2004). Under a particular configuration of the Dirichlet process, a collection of $K$ amino acid fitness profiles (designated $\psi=\left(\psi_{l}^{(k)}\right)_{1 \leq l \leq 20}^{1 \leq k \leq K}$ with the constraint $\sum_{1 \leq k \leq 20} \psi_{l}^{(k)}=1$ for all $k$ ), are used to define a selection coefficient, used to modulate nonsynonymous rates. The profile allocated to any codon site $n$ is denoted $z_{n}$. In other words, $z_{n}$ returns an index from 1 to $K$ corresponding to the profile operating at site $n$. The selection coefficient associated with the amino acid replacement in going from codon $i$ to $j$ at site $n$ is given as $S_{i j}^{(n)}=\ln \psi_{f(j)}^{\left(z_{n}\right)}-\ln \psi_{f(i)}^{\left(z_{n}\right)}$, where $f(i)$ returns an index from 1 to 20 based on the amino acid encoded by codon $i$. Altogether, the codon substitution matrix of the model is given as:

$$
Q_{i j}^{(n)}= \begin{cases}0, & \text { if the codons differ by more than one position, } \\ \rho_{i_{c} j_{c}} \varphi_{j_{c}}, & \text { if synonymous, } \\ \rho_{i_{c} j_{c}} \varphi_{j_{c}}, \frac{S_{i j}^{(n)}}{1-e^{-S_{i j}^{\left(z_{n}\right)}}} & \text { if non-synonymous. }\end{cases}
$$

Later in this work, we shorten this notation to $\mu_{i j}=\rho_{i_{c} j_{c}} \varphi_{j_{c}}$

Rodrigue et al. (2010) found that using a Dirichlet process to infer the amino acid fitness profiles is computationally intensive. To address this, they introduced models directly utilizing profiles from the C20, C40 and C60 finite empirical mixture models in Quang et al. (2008), dubbing them MG-MutSelC20, MG-MutSelC40, and 
MG-MutSelC60. Bayes factors indicated that these models outperformed traditional codon models, and so we have used MG-MutSelC60 in our work in the next chapter.

\subsubsection{Preference Shifts and Time-Heterogeneity}

All the models described above make the simplifying assumption that the substitution process is time-homogeneous, that is, the underlying substitution mechanisms do not vary along the branches of the tree. However, this is unrealistic, and many violations of this assumption are known.

Some examples of changes in the substitution process include shifts in the fitness landscape of protein-coding sequences over a phylogenetic tree due to purifying and diversifying selection effects. These shifts could be characterized by a change in "preference" for specific amino acids in homologous sites, in related species or taxa. Some examples of these shifts include changes to the external environment, such as historical changes in the gas composition in oceans over the eons or transitioning from aquatic environments to land-based environments. Changes to the inter- or intracellular environment in which the protein functions can also bring about preference shifts, such as changes in antibody configuration in bacterial and viral hosts.

One well-documented example is the avian-human host-shift event in the influenza A virus (Dos Reis et al., 2010). Tamuri et al. (2009) examined avian-human hostshifts, as shown as differences in site-specific amino acid preference shifts across subclades of avian- and human-host influenza strains. These shifts are directly related to changes in the antigenic sites of the hemagglutinin and neuraminidase proteins, which are required for the virus to bind to the host cell Baigent and McCauley, 2003), and which show marked receptor specificity in human hosts (Webster et al., 1992). The influenza polymerase complex of PA, PB1 and PB2 also interacts with host factors and shows host specificity (Taubenberger et al., 2005). These genes form a heterotrimer which is involved in cap-snatching and initiation of transcription in 
the host, and a residue change from glutamic acid to lysine in PB2 site 627 is strongly correlated with human-host virulence (Nilsson et al., 2017). We investigate the case of these viral host-shift events in the next chapter. 


\section{Chapter 2}

\section{Detecting Amino Acid Preference Shifts with Codon-Level Mixture Models}

\section{$2.1 \quad$ Introduction}

Detecting shifts in site-specific amino acid preferences across species or strains poses a number of technical challenges (Bazykin, 2015). Some early approaches relied on information-theoretic calculations, performed on sequence alignments directly (Chen et al., 2006; Finkelstein et al., 2007; Miotto et al., 2008). These methods assume that the molecular sequence of each species or stain in an alignment provides an independent set of observations across all sites. Quite often, however, the sequences of an alignment under analysis are in fact closely related to one another, and methods that fail to account for these relations are susceptible to numerous types of problems (Bruno, 1996). Indeed, the issue of non-independence of sequences in a sample is what drives the field of phylogeny-based methods of analysis (Felsenstein, 2004; Yang, 2014 ). 
In keeping with this understanding, phylogenetic approaches that account for shifts in amino acid preferences have been explored, with perhaps the most sophisticated of these being the CAT-BP model (Blanquart and Lartillot, 2008). Its name is meant as a shorthand for a model which, in effect, attempts a CATegorization across sites of amino acid profiles, with break-points along the tree that make changes to the amino acid profiles governing each site. Realizations of the substitution process are thus heterogeneous across the positions of an alignment, and along the branches of the phylogeny, with both types of heterogeneity inferred directly from the data. While the principles of the CAT-BP model are attractive, the time-heterogeneity of amino acid composition is modulated globally across all sites, which is a different perspective than one seeking to uncover particular sites having undergone changes in amino acid profiles. Moreover, the model is difficult to work with in practice, given the elaborate set of Monte Carlo operators involved in its implementation, and more work is needed to make the CAT-BP model tractable for larger data sets.

Roure and Philippe (2011) investigated an approach building solely on the CAT model (Lartillot and Philippe, 2004), with a multi-stage system aimed at testing specific hypotheses about the time-heterogeneity of amino acid profiles across a few sub-clades of a phylogenetic tree. In their approach, a first Markov chain Monte Carlo $(M C M C)$ run is conducted with the CAT model on a dataset of interest. A post-treatment of this first MCMC is done to define a finite set of profiles that are meant to be an approximation of the distribution of amino acid profiles across sites. Using the so-defined empirical finite mixture model, a second set of MCMC runs is conducted, with each run taking place on a particular sub-set of the original data, in order to obtain an estimate of each site's allocation probability to each of the components of the empirical mixture. A post-treatment of this second set of MCMC runs is performed on the basis of site allocation probabilities in each data sub-set, so as to compute the probability of identical profiles $(P I P)$ across data sub-sets for 
each site. A low PIP signals a potential candidate of a shift in amino acid preference across data sub-sets. But a strictly amino acid-level formation as used by Roure and Philippe (2011) relinquishes the mechanistic formulation (Halpern and Bruno, 1998) that allows for the analyses to be conducted directly on the protein-coding DNA sequence data, in a joint estimation of several features of the evolutionary process.

Codon-level approaches, relying on the mutation-selection framework (Halpern and Bruno, 1998; Yang and Nielsen, 2008) have more recently been applied to the modelling of changes in amino acid profiles, either with site-specific-clade-specific maximum likelihood estimation (Tamuri et al., 2012, 2014; Dos Reis et al., 2016), which may sometimes run the risk of over-parameterization (Rodrigue, 2013); with experimentally derived profiles (Doud et al., 2015), which are only applicable for few genes; or with hierarchical Bayesian methods (Parto and Lartillot, 2017), which intrinsically treat across-site variation as random-effects, and in this case account for across-time variation over specific clades of interest. Again, the computational challenges from the latter approach are significant, and the richness of the differential selection profile parameterization seems to leave it with low power (Parto and Lartillot, 2017).

Here, we adopt a method that combines some of the ideas proposed in Roure and Philippe (2011) with the mutation-selection approach described in Rodrigue et al. (2010). The method operates with a predefined finite mixture of amino acid profiles, which are introduced into the mutation-selection codon substitution framework (Rodrigue et al., 2010; Rodrigue and Aris-Brosou, 2011), and uses MCMC to compute the PIP across different sub-sets of sequences from the data, corresponding to different parts of a phylogenetic tree. Using simulations in a realistic set of conditions, we find that the method has good power, with a low false-positive rate, when the shifts in amino acid profiles correspond to marked differences in profiles. However, when the distinctiveness of profiles is low across the sub-tree of interest compared to the 
rest of the tree, the method performs more poorly. Our simulations illustrate one way of studying how the method's performance changes for different levels of profile distinctiveness. Analysis on real data shows results consistent with previous methods, with some notable exceptions.

\subsection{Materials and Methods}

\subsubsection{Data}

We used the Influenza PB2 gene alignment assembled by Tamuri et al. (2009), comprised of 321 avian-host and 80 human-host strains. The reference tree topology was also taken from Tamuri et al. (2009) and was invariant throughout the analysis. This tree is structured such that human-host host sub-tree is monophyletic, as shown in figure 2.1 (Tamuri et al., 2009). We are therefore focused on detecting shifts that may have occurred in human-host strains, following the transfer from avian hosts. 

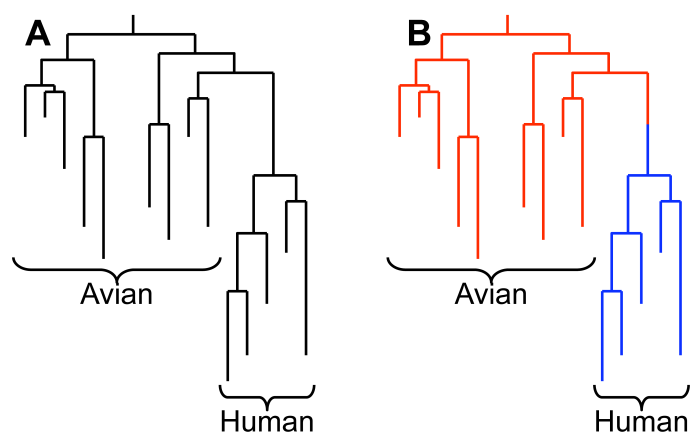

Figure 2.1: Influenza PB2 phylogenetic tree, with monophyletic human-host clade. Taken from Tamuri et al. $(2009)$

\subsubsection{Model}

The codon-level substitution model we use follows the mutation-selection framework (Yang and Nielsen, 2008; Halpern and Bruno, 1998; Rodrigue et al., 2010). At codon site $n$, the entries in the $61 \times 61$ matrix $Q^{(n)}$, specifying the infinitesimal rate from codon $i$ to codon $j$, are given as:

$$
Q_{i j}^{(n)}=\mu_{i j} \frac{S_{i j}^{(n)}}{1-e^{-S_{i j}^{(n)}}}
$$

where $\mu_{i j}$ controls the nucleotide-level mutational process, and $S_{i j}^{(n)}$ is the (scaled) selection coefficient associated with going from the amino acid encoded by $i$ to that encoded by $j$ at site $n$ (Rodrigue et al., 2010). This selection coefficient is obtained from $S_{i j}^{(n)}=\ln \psi_{f(j)}^{\left(z_{n}\right)}-\ln \psi_{f(i)}^{\left(z_{n}\right)}$, where $\psi^{\left(z_{n}\right)}$ is the amino acid profile allocated to site $n$, $\ln \psi_{f(i)}^{\left(z_{n}\right)}$ is the (scaled) fitness of the amino acid encoded by codon $i$ at that site, $f(i)$ returns an index from 1 to 20 based on the amino acid encoded by codon $i$, and $z_{n}$ is an auxiliary variable returning an index, from 1 to $K$, specifying the allocation of site $n$ to component $k(1 \leq k \leq K)$. Our Markov chain Monte Carlo sampler performs updates on $z$, along with other parameters collectively denoted as $\theta$, but we focus our description on the update mechanisms of the former since the approach we utilize is 
seldom discussed in the contexts of phylogenetic finite mixture models. Assuming an initial random allocation has been set, a Gibbs update for the allocation of a particular datum, denoted as $D_{n}$, proceeds through the following steps: First, supposing that the datum allocation being subjected to the update is currently set to component $k$, i.e., $z_{n}=k$, we decrease by 1 the count of the number of data columns affiliated to that component, denoted $\eta_{k}$. Then, among the $K$ possible components of the mixture, we draw a new $k$, and set $z_{n}=k$, with probability $\propto\left(\eta_{k}+1\right) p\left(D_{n} \mid \theta, \psi^{(k)}\right)$. The auxiliary variable approach to our sampler is a form of demarginalization or parameter expansion (Rodrigue et al., 2007), with respect to approaches that operate with weighted sum likelihood function at site $n$ :

$$
p\left(D_{n} \mid \theta\right)=\sum_{k=1}^{K} w_{k} p\left(D_{n} \mid \theta, \psi^{(k)}\right)
$$

where $w=\left(w_{k}\right)_{1 \leq k \leq K}$ (with the constraint $\sum_{1 \leq k \leq K} w_{k}=1$ ), is a weight vector with $w_{k}$ being the prior probability of a given site being allocated to component $k$. Updating as we do implicitly integrates over the weights, and is equivalent to having a flat Dirichlet on them (Robert and Casella, 2010).

Here, our mixture models are based on two predetermined sets of amino acid profiles. First, we arbitrarily defined a set of profiles (which we denote "MutSelBC") based loosely on a grouping of side chain biochemical properties:

- Small nonpolar: alanine, glycine, serine, threonine

- Aromatic: phenylalanine, tryptophan, tyrosine

- Nonpolar aliphatic: isoleucine, leucine, valine, methionine

- Polar positive: histidine, lysine, arginine

- Polar negative: aspartic acid, glutamic acid 
- Polar neutral: asparagine, glutamine

- Proline

- Cysteine

This grouping into eight profiles was selected to have no overlap in amino acid residues, so as to construct the most biologically obvious shifts in amino acid preferences. We controlled the intensity of purifying selection against amino acids excluded from a group by distributing the probability mass of a profile mainly to the members of a group as explained in the opening of the results section.

As a second alternative, we used the C60 mixture model profiles from Quang et al. (2008), which were derived from an amino-acid-level maximum likelihood analysis of a large set of empirical data. As in Rodrigue et al. (2010) and Rodrigue and ArisBrosou (2011), we refer to this model as MutSelC60.

\subsubsection{Probability of Identical Profiles}

Using preset values for our mixture models, in conjunction with our MCMC sampling methods, allows for straightforward calculations of the probabilities of each site of an alignment belonging to each component of the mixture. Specifically, from a collection of draws of $z$ from the posterior probability distribution obtained via MCMC, we calculate the probability of allocation of site $n$ to component $k$ as simply the proportion of draws where $z_{n}=k$ in our sample, which we denote as $p^{(n)}(k)$. The same procedure can be applied for an analysis where the allocation of site $n$ is (potentially) different in two sub-trees, in this case giving us the probability of allocation of site $n$ to component $k$ in the human-host sub-tree $p_{\text {hu }}^{(n)}(k)$, and that of the avian-host sub-tree $p_{\mathrm{av}}^{(n)}(k)$. In this latter context, the probability of identical profiles Roure 
and Philippe, 2011) at site $n$ is calculated as follows:

$$
P I P_{n}=\sum_{k=1}^{K} p_{\mathrm{hu}}^{(n)}(k) \times p_{\mathrm{av}}^{(n)}(k)
$$

Note that $P I P_{n}=1$ for a site with $100 \%$ probability of allocation to the same profile across the two sub-trees and $P I P_{n}=0$ if the allocation is entirely to different profiles across the two sub-trees.

\subsubsection{Simulations}

We simulated data alignments using the posterior mean nucleotide-level parameter values obtained when running the MutSelC60 model (Rodrigue et al., 2010), as well as branch lengths and several different amino acid profiles, as described above. Some simulations consisted of running the same substitution process over the entire tree (comprising both human- and avian- host strains), which can be viewed as the control simulations, whereas others used different amino acid profiles in the avian- and human-host sub-trees.

These simulations and calculations were conducted in a modified version of PhyloBayesMPI 1.7 (Lartillot et al., 2013; Rodrigue and Lartillot, 2014), which outputs allocation probabilities for MCMC runs under finite mixture models.

\subsection{Results and Discussion}

\subsubsection{Simulation Data}

We first evaluated our approach using these simulations. With the exception of amino acid profiles, we generated data from parameter values obtained from the posterior mean of an analysis using a mutation-selection model (Rodrigue and Aris-Brosou, 2011) on an alignment of the influenza PB2 gene (Tamuri et al., 2009). This dataset 
mainly consists of avian-host strains but also includes a monophyletic group of humanhost strains, and some simulations thus used a different set of amino acid profiles in the human-host sub-tree than in the rest of the (avian-host) tree. We explored simulations under several sets of amino acid profiles. The first set, which we denote "MutSelBC", is arbitrarily defined, and based loosely on side chain biochemical properties. These profiles were selected to have no overlap in the dominant amino acid residues, such that biologically clear preference shifts at a given position would be represented as profile shifts coinciding with the host transition (Tamuri et al., 2009).

Figure 2.2 depicts four versions of the eight MutSelBC profiles that we used for our first series of simulations. In these logoplots, the height of each letter in the column represents the probability mass for that amino acid residue in the profile. These four profile sets show a gradient in what we refer to as peakedness. We use this term to refer to the probability mass that we distribute equally to the amino acids of a profile group, with the complement being distributed equally to the remaining amino acids not part of that group. The first profile set shows a $50 \%$ peakedness value (leftmost panel of figure 2.2. . Thus, in the first profile in this set, valine, methionine, leucine and isoleucine together have a 50\% probability mass, while all other amino acids account for the other $50 \%$, the second profile in the set has $50 \%$ distributed evenly to tyrosine, tryptophan, and phenylalanine, with the 17 other amino acids splitting the remaining 50\%, and so on. The three other panels of figure 2.2 show profiles with dominant amino acids sharing a probability mass of $75 \%, 90 \%$, and 99.9\%. The peakedness parameter provides a crude means of controlling the intensity of the constraint for the amino acids of a group. 


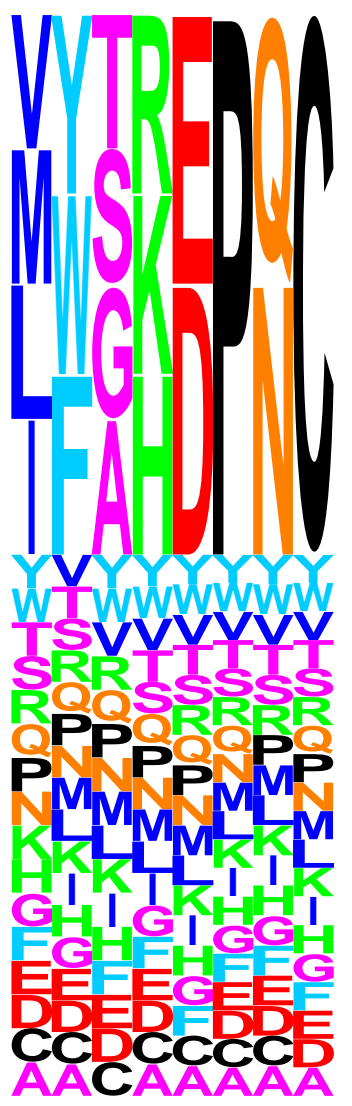

0.50

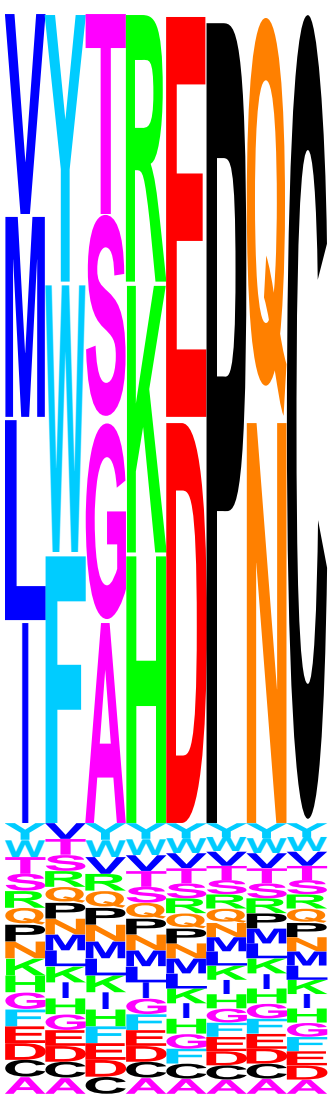

0.75

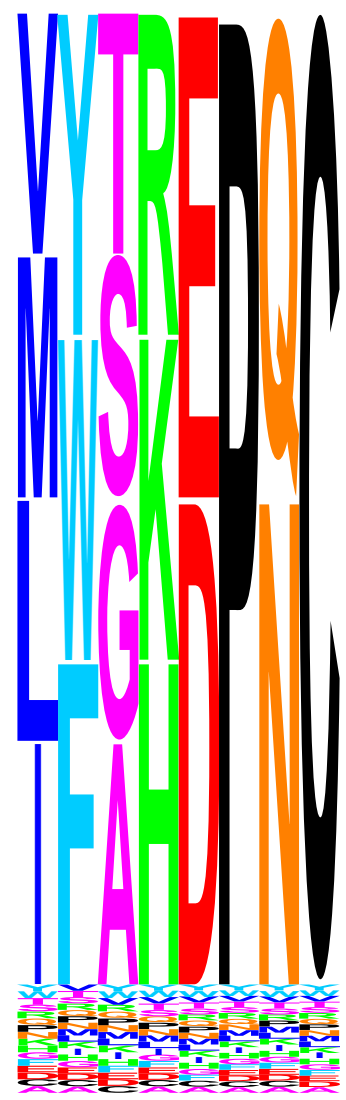

0.90

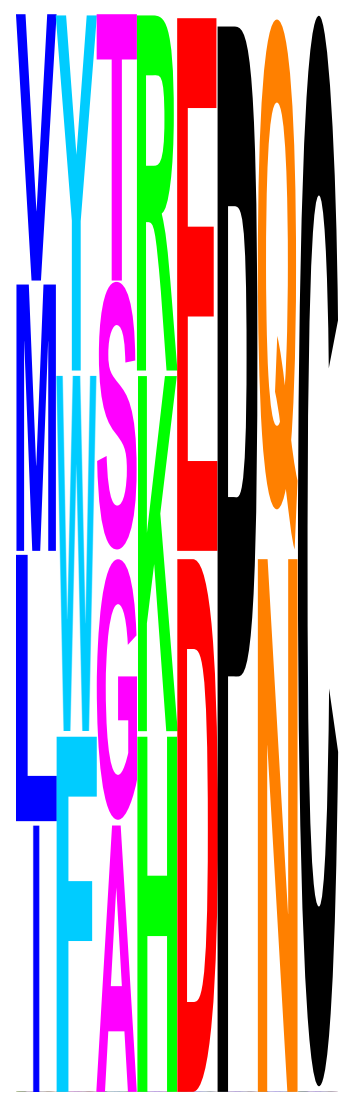

0.999

Figure 2.2: Logoplots of MutSelBC profile sets with four different "peakedness" values, indicating probability mass of the chosen amino acids in each profile. For each profile set, the probability of the profile-specific amino acid is 50\%, 75\%, 90\% and $99.9 \%$ respectively.

The effect of the peakedness of the simulation profiles on the subsequent analyses can be seen in the logoplot in figure 2.3. This figure displays the allocation probability of a particular site, simulated with the first profile at $90 \%, 75 \%$ and $50 \%$ peakedness. These simulations respectively amount to a relatively strong, moderate, and weak selection constraint for nonpolar aliphatic residues at that site. As the peakedness decreases, the allocation probability to the profile used to simulate can be seen to decrease. In other words, when the selection constraint for a particular group of amino acids is weak, so is the allocation probability. 

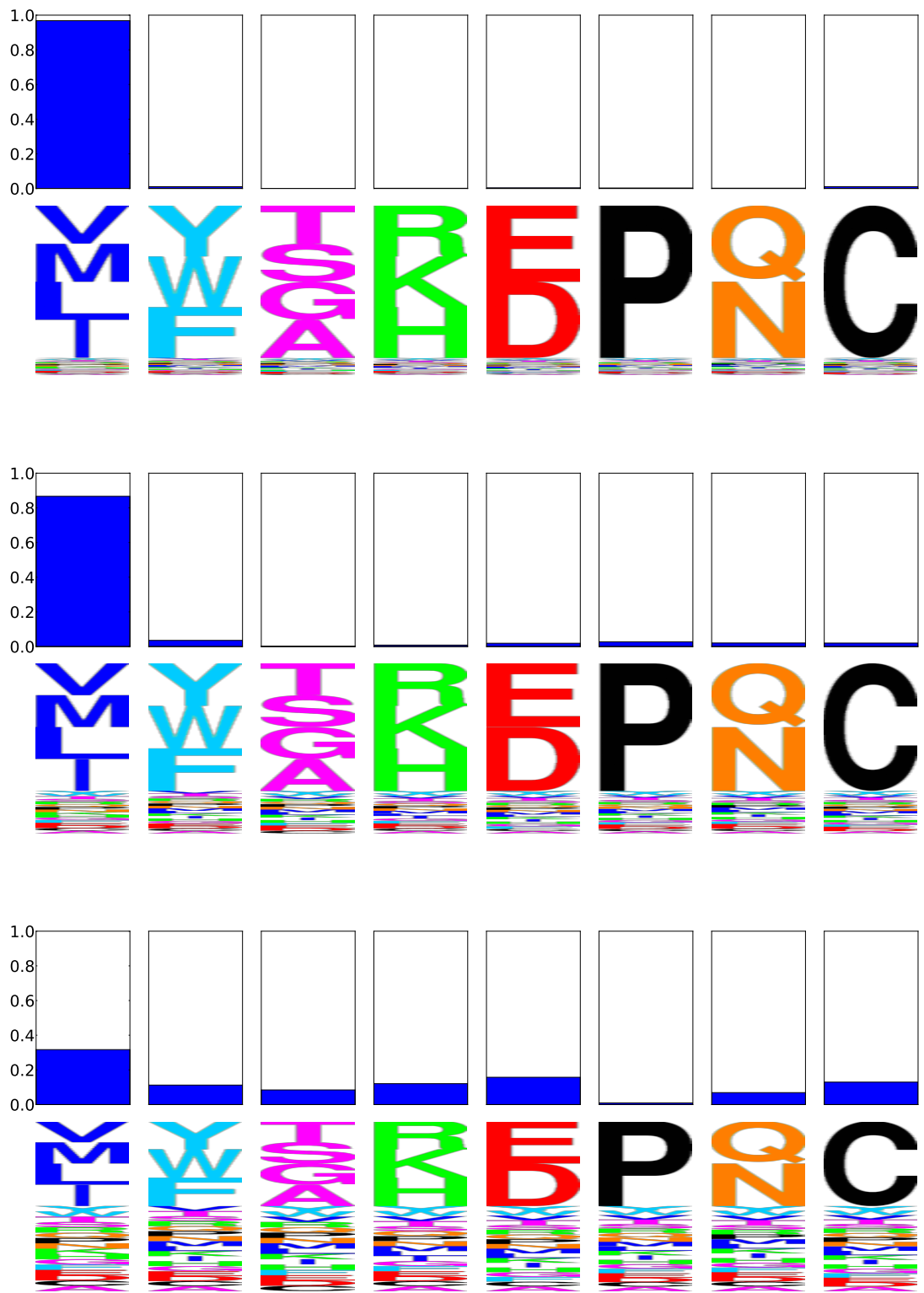

Figure 2.3: Allocation probability logoplots for the same codon site using MutSelBC profiles with $90 \%$ (top), $75 \%$ (middle) and 50\% (bottom) "peakedness" values. For each logoplot, the solid bar indicates the allocation probability to a given profile at that site, and the letters underneath indicate the profile. The letters are scaled to indicate the probability of that amino acid at the site in that profile.

Figure 2.4 summarizes the broader implications of profile peakedness on the simulation results. Alignments were simulated as before, with a peakedness ranging from 0.50 to 0.999 . For each alignment, 80 of the 759 codon sites were evolved under a 
different profile regime in the human-host sub-tree than in the rest of the tree, to simulate a distinct preference shift at those sites, corresponding to a host shift. The remaining sites were evolved under the same profiles for the entire tree. We then analyzed these alignments by evaluating the probability of identical profiles (PIP) at each site across the avian- and human-host sub-trees.

A receiver operating characteristic (ROC) plot of sensitivity vs specificity for the range of PIP thresholds between 0.00 and 1.00, for each of the above simulated data sets (figure 2.6) was performed. A PIP at or below 0.05, in which $95 \%$ of the probability mass was allocated to different profiles between clades, was found to be suitably discriminating at all peakedness values. This threshold was used as the definition of a preference shift between clades.

It can be seen that the true-positive detection rate, that is, the ability to correctly identify the sites that were evolved with different amino acid profiles across the two sub-trees by having a low PIP score, increased dramatically as a function of the peakedness of the profiles (top left). A peakedness of 0.999 identified $100 \%$ of the embedded profile shifts. In other words, unsurprisingly, the approach is highly effective in detecting amino acid profile which are markedly different in the two sub-trees.

True negative detection, that is, the correct identification of non-shifted sites via a high PIP score, was mostly consistent among all peakedness values (top right). This is as expected, as the MutSelBC profiles were constructed to be clearly distinguishable from each other, particularly at high peakedness, where the potential for overlap in residues visited would be minimal.

False positive detection, where PIP was low despite there not being an embedded shift, was low for all peakedness levels. Profiles with 0.95 and 0.999 peakedness were markedly lower in false positives, with less than $5 \%$ and $0 \%$ respectively. Again, at this level of peakedness, the profiles are fairly rigid and there is little or no overlap of amino acids across the set of profiles. 
False negatives, where PIP was high despite there being an embedded shift, also showed a direct relationship to the peakedness of the profiles (bottom right). This is likely due to the lower peakedness profiles being less distinct from each other, simulating a weak allocation.

Figure 2.5 shows the the results of simulation analysis for C10, 20, 40, and 60 profiles. All of these profiles showed considerably higher false positive rates compared to the MutSelBC profiles, with $\mathrm{C} 10$ the least at $12.5 \%$ and $\mathrm{C} 60$ the most at $60.7 \%$. True positive rates were generally lower than MutSelBC, ranging from $60.6 \%$ for $\mathrm{C} 10$ to $91.8 \%$ for $\mathrm{C} 60$. 


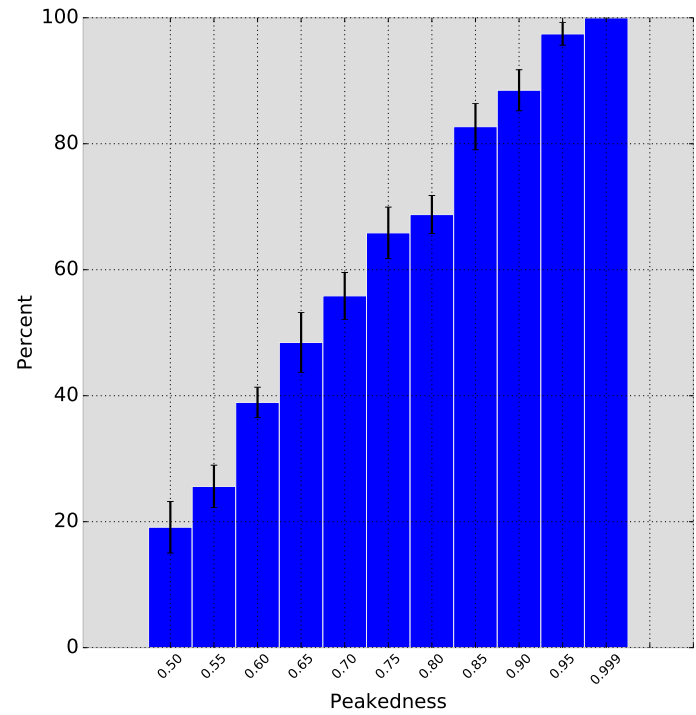

(a)

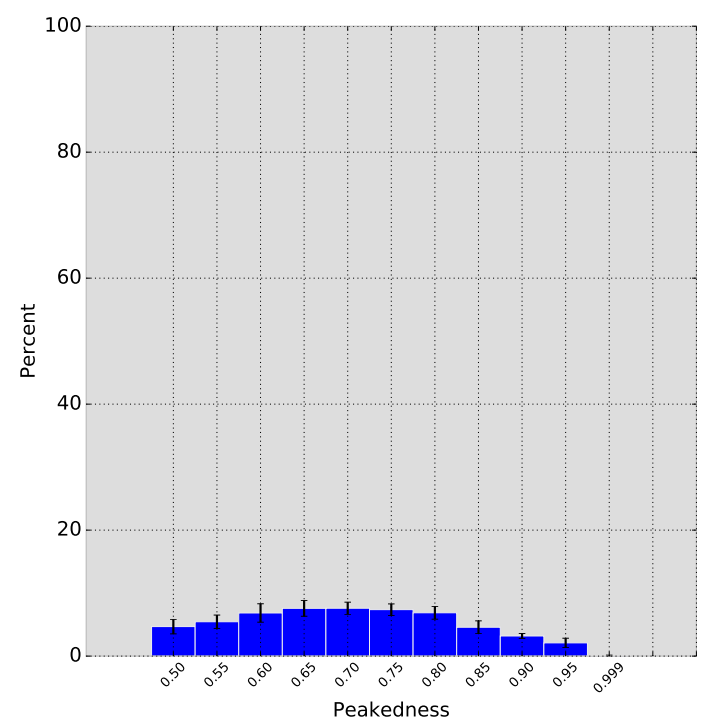

(c)

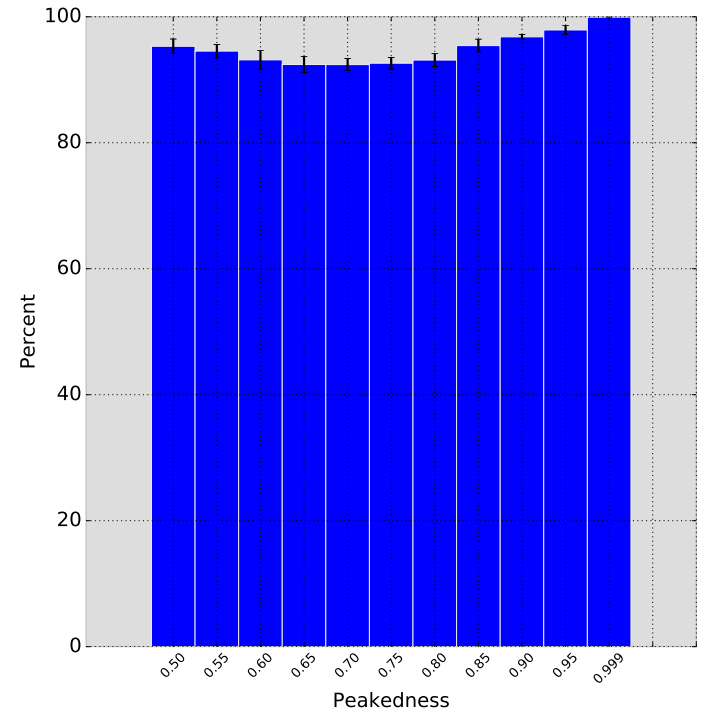

(b)

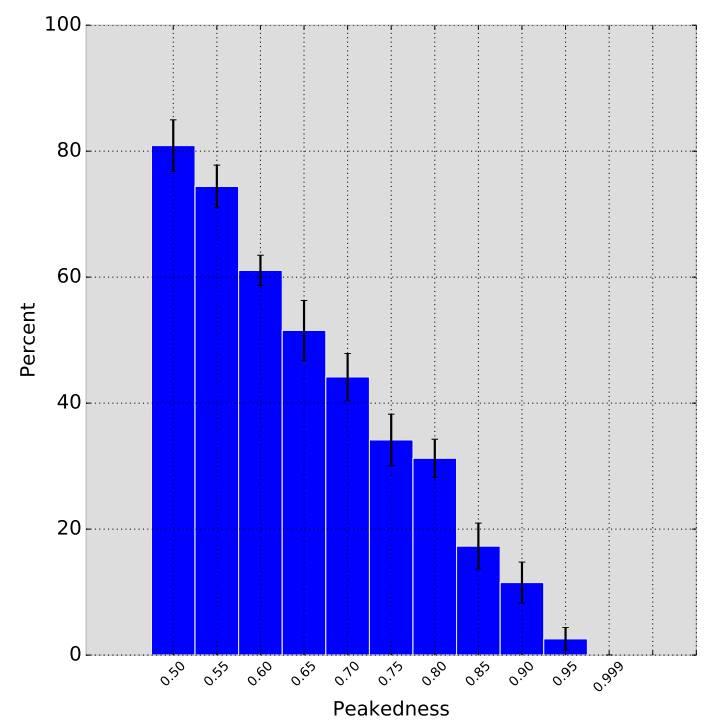

(d)

Figure 2.4: (a) True positive, (b) true negative, (c) false positive and (d) false negative detection of preference shifts, by peakedness value, for simulated data using MutSelBC profiles. 


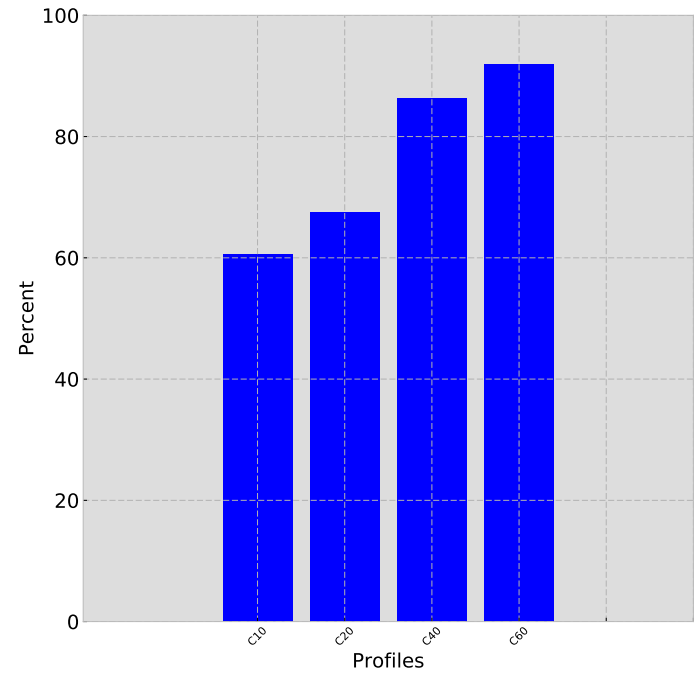

(a)

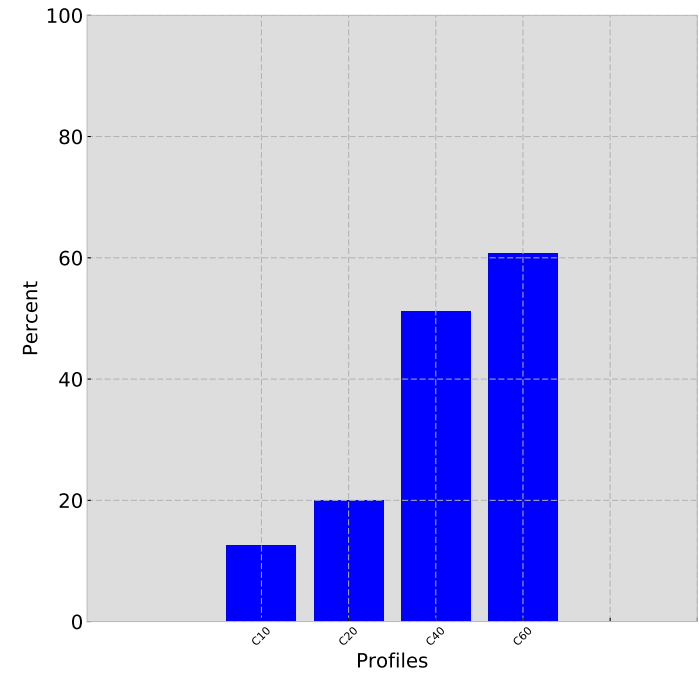

(b)

Figure 2.5: (a) True positive, and (b) false positive detection of preference shifts value, for simulated data using C10, C20, C40 and C60 profiles. 


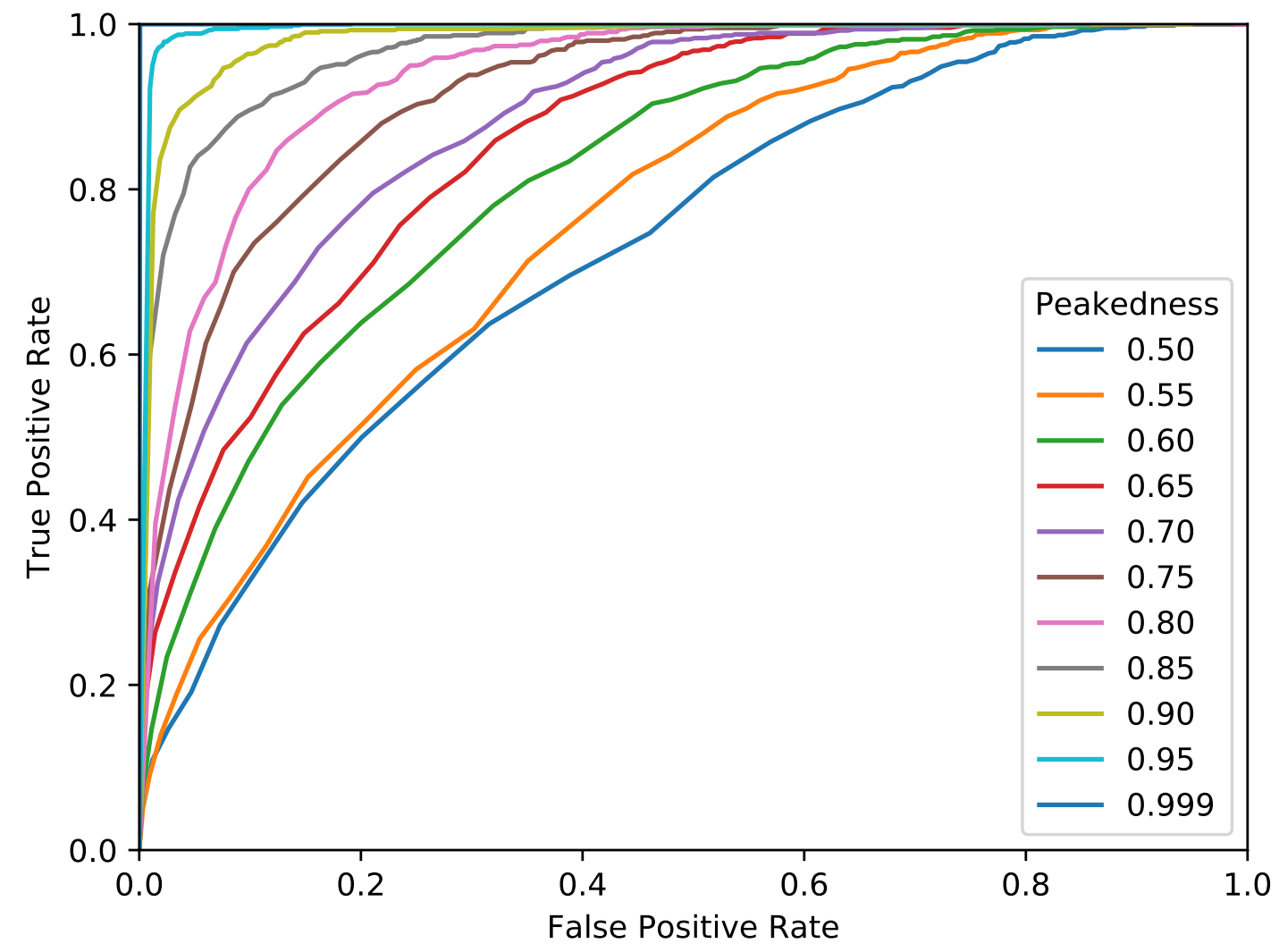

Figure 2.6: Receiver operating characteristic plot for PIP thresholds used to define a preference shift. Data was simulated with peakedness values ranging from 0.50 to 0.999. A PIP threshold of 0.05 was determined to acceptably encompass preference shifts in all peakedness values. 


\subsubsection{Real Data}

Figure 2.7 is a demonstration of a preference shift between avian (red) and human (blue) strains as detected by the MutSelC60 (top) and MutSelBC (bottom) profiles. However, closer examination of the profile allocations in MutSelC60 indicates that the apparent shift may be an artifact of the ambiguity of the profiles. In the avianhost strains, the site appears to allocate primarily to three different profiles, but all three of these profiles show preference for small nonpolar amino acids (ASTG). The human-host strains almost entirely allocated to a single profile, which although different from the avian profiles, also shows preference for small nonpolar side chains (AST). There may be little functional difference, or indeed no difference in residues at the site, but different weighting of the same residues in different profiles gives a false positive preference shift detection. The MutSelBC profiles at bottom, which have a single profile for small nonpolar side chains (TSGA), show almost no difference in profile allocation between the two clades.

Figure 2.8 shows allocation to MutSelBC profiles for sites with high and low PIP scores. In the top panel, the avian and human clades allocated strongly to the same profiles ( $\mathrm{PIP}=0.865$ ), while the middle panel shows the case where both clades allocated completely to different profiles $(\mathrm{PIP}=0.00)$. The bottom panel has a relatively low PIP score (0.115), but this is due to weak allocation to any profile, in both clades, rather than the result of a clear preference shift at that site.

Table 2.1 shows PIP scores for sites using C60 and MutSelBC profiles. Sites that were identified in Finkelstein et al. (2007) as being significantly shifted between human and avian clades are also listed, along with the euclidean distance of their amino acid frequency vectors. Sites identified in Tamuri et al. (2009) as displaying a preference shift between human and avian clades are also listed.

Tamuri et al. (2009)'s methodology in measuring the magnitude of the preference 

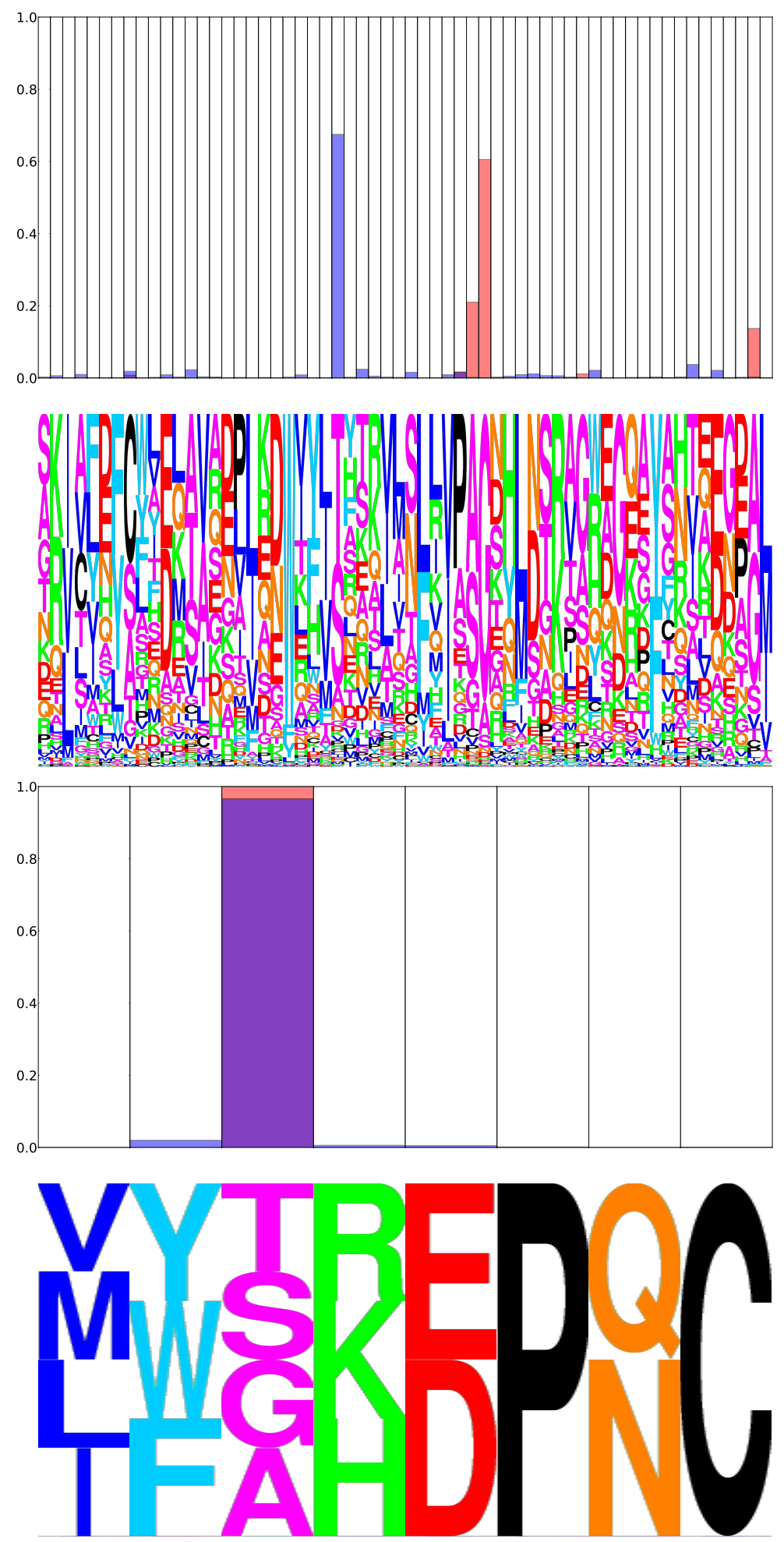

Figure 2.7: Allocation probability logoplot using MutSelC60 (top) and MutSelBC (bottom) profiles for codon site 661. Red is avian-host strains and blue is humanhost strains of Influenza PB2. Purple indicates overlapping profile allocation in both clades. 


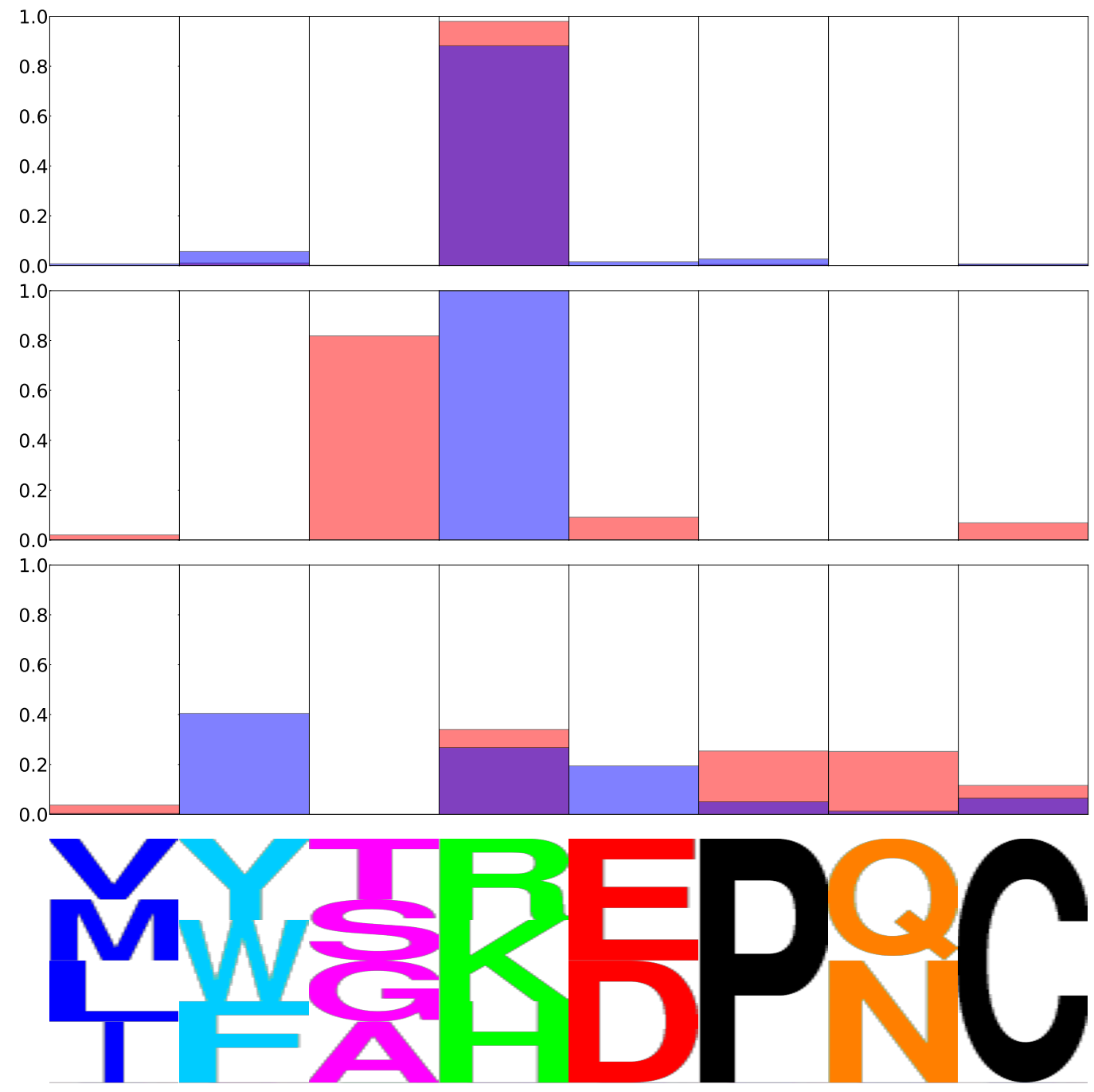

Figure 2.8: Allocation probability logoplots in avian (red) and human (blue) strains of Influenza PB2. Purple indicates overlapping profile allocation in both clades. At top is a strongly identified preference shift with a PIP of 0.865. At middle is a strongly identified non-shift, with a PIP of 0.00 . At bottom, the low PIP of 0.115 does not indicate a preference shift, as site is weakly allocated to a number of profiles in both clades. 


\begin{tabular}{|c|c|c|c|c|}
\hline Site & PIP (MutSelC60) & PIP (MutSelBC) & Finkelstein & Tamuri \\
\hline 44 & 0.0582 & 0.2155 & 0.966 & YES \\
\hline 64 & 0.148 & 0.0170 & 0.954 & \\
\hline 76 & 0.00346 & 0 & & \\
\hline 81 & 0.0165 & 0 & & \\
\hline 102 & 0.166 & 0.0000160 & & \\
\hline 105 & 0.00535 & 0 & & YES \\
\hline 106 & 0.0184 & 1 & & \\
\hline 107 & 0.0116 & 0.00637 & & \\
\hline 109 & 0.272 & 0.0005 & & \\
\hline 122 & 0.0708 & 0.00608 & & \\
\hline 199 & 0.0278 & 1 & 0.997 & YES \\
\hline 249 & 0.445 & 0 & & \\
\hline 271 & 0.0154 & 0.974 & 0.958 & \\
\hline 292 & 0.0127 & 0.0265 & & \\
\hline 338 & 0.0124 & 0 & & \\
\hline 377 & 0.00150 & 0.0655 & & \\
\hline 471 & 0.00383 & 0.0845 & & \\
\hline 475 & 0.690 & 1 & 0.994 & YES \\
\hline 493 & 0.00257 & 0.91 & & YES \\
\hline 522 & 0.0516 & 0.008 & & \\
\hline 524 & 0.168 & 0.00547 & & \\
\hline 559 & 0.00799 & 0.260 & & \\
\hline 567 & 0.0852 & 0.216 & 0.977 & \\
\hline 569 & 0.175 & 1 & & YES \\
\hline 588 & 0.00283 & 0.00609 & 0.971 & \\
\hline 591 & 0.265 & 0.001 & & \\
\hline 613 & 0.0776 & 0.115 & & YES \\
\hline 627 & 0.0111 & 0 & 0.977 & YES \\
\hline 661 & 0.00126 & 0.967 & & YES \\
\hline 674 & 0.00644 & 0.0555 & 0.969 & \\
\hline 676 & 0.0165 & 0.242 & & \\
\hline 682 & 0.372 & 0 & & YES \\
\hline 684 & 0.0596 & 0.999 & & YES \\
\hline 702 & 0.552 & 1 & 0.955 & YES \\
\hline 711 & 0.170 & 0 & & \\
\hline 715 & 0.0916 & 0.00584 & & \\
\hline 740 & 0.00239 & 0 & & YES \\
\hline 754 & 0.0125 & 0.0335 & & \\
\hline
\end{tabular}

Table 2.1: Codon sites with preference shifts detected with MutSelC60 and MutSelBC profiles, and sites identified in previous studies (Finkelstein et al. (2007) and Tamuri et al. (2009) ). 
shifts makes it difficult to compare findings, but several cases exist where the shift as detected by Tamuri et al. (2009) were to a functionally similar profile in MutSelBC. We illustrate several examples below.

Site 44 was allocated to alanine-dominant profiles in the avian clade and serinedominant profiles in human, albeit with very weak allocation in the human clade. Tamuri identified an alanine preference in the avian clade and a leucine preference in human, but both had a strong secondary preference for serine.

Site 475 allocated strongly to leucine-dominant profiles in the avian clade, and methionine-dominant in human. Tamuri found similar results, with avian preferring leucine with a secondary preference for methionine, while human preferred methionine only.

Site 569 strongly allocated to threonine-dominant profiles in the avian clade, while the human clade was spread between threonine, alanine, serine and glycine-dominant profiles. Tamuri identified threonine (secondary alanine) in avian, and alanine (secondary serine) in human.

Site 613 showed weak allocation in both clades, with avian favouring valinedominant profiles and human split between valine, methionine and leucine. Tamuri identified valine with secondary alanine and isoleucine for avian, while human was threonine, with secondary isoleucine and alanine.

Site 702 strongly allocated to lysine-dominant profiles in the avian clade and arginine-dominant profiles in human. Tamuri identified avian as lysine with secondary arginine, and human as arginine.

As can be seen, instances where neither MutSelC60 or MutSelBC profiles agreed with Tamuri et al. (2009) were largely due to the biochemical similarity of the residues involved, which caused allocation to similar or identical profiles, or due to Tamuri et al. (2009) identifying a change in proportion of preference to the same amino acids between clades, which was not considered a functional shift under finite mixture 
models.

\subsection{Conclusion}

We can see that finite mixture models are capable of effectively detecting preference shifts in simulated viral sequence alignments, especially where the profile shift is highly pronounced. This is the case for MutSelBC profiles with a high peakedness value: inferences become progressively less powerful as shifts become less prominent. However, these profiles are arbitrarily defined and relatively crude, with equal probability mass given to all residues in a profile.

C60 profiles, which are more objectively constructed from empirical data, show a middle ground in effectiveness of preference shift detection. However, one drawback of using this mixture model is the allocation of sites to similar profiles, registering as a profile shift and resulting in false positives, as detailed in the discussion for table 2.1 above. This raises the question of whether profile shifts between biologically similar residues truly represent an adaptive shift. After all, empirical amino acid matrices such as LG (e.g., Le and Gascuel, 2008) are based on the rationale that some pairs of amino acids are highly exchangeable, and may be nearly equivalent in fitness. It may be that statistically significant shifts may have low biological significance.

The MutSelBC model is blind to these types of shifts. For example, A199S is detected as a host shift marker by MutSelC60, Finkelstein et al. (2007) and Tamuri et al. (2009), but is contained in the same profile and considered strictly equivalent by MutSelBC, and could never be detected with this model. Model assessment for C20, C40 and C60 profiles were evaluated in Rodrigue et al. (2010) by computing Bayes factors, and were found to outperform homogeneous mutation-selection models, with increasing performance as the richness of the profile mixtures increased. We would assume that the MutSelBC Bayes factors would naturally be a lower fit than the $\mathrm{C}$ 
series. The C20, C40 and C60 models were later found to outperform models with an amino acid profile for every site (Rodrigue and Aris-Brosou, 2011).

It is also important to take the entropy of the allocation probability vector of a site into account when using these methods, which may be affected both by the strength of the constraint at that site, and the phylogenetic signal available in the sequence alignment. A large variation in profile allocation within taxa in a clade can easily be erroneously identified as a preference shift between clades, as the weakly-allocated clade will register as having multiple profiles, distinctly contrasting with a clade that is strongly allocated to one profile.

Important improvements could be realized by using empirical profiles constructed within the codon mutation-selection context, rather than MutSelC60 which was originally derived in an amino-acid replacement context. Ideally, we could have a model that simultaneously derives profiles (Blanquart and Lartillot, 2008) while also jointly inferring changes in the profiles at specific site, at specific branches in the tree. We develop some of the ideas in the next chapter. 


\section{Chapter 3}

\section{Future Directions}

\subsection{Empirical Profiles in a Mutation-Selection Con- text}

The MutSelBC profiles have been chosen arbitrarily according to characteristics of the residue side chains and are not directly informed by empirical data. A further refinement would be to derive profiles via a maximum-likelihood algorithm similar to Quang et al. (2008). Quang et al. (2008)'s profiles were developed with an aminoacid level model, where the frequency vectors were subject to the joint effects of mutation and selection. This could be less appropriate for our codon-level model, which accounts for mutation and selection effects separately. A valuable investigation to undertake would be to run an expectation-maximization algorithm with our mutation-selection model on the same data set as was used for Quang et al. (2008), which, if sufficiently different, would indicate that the new set of profiles would be worthwhile in our context. 


\subsection{Nonstationary Substitution Models}

Though the codon-level mutation-selection model addresses site heterogeneity in coding sequences, like most substitution models it assumes a stationary Markovian substitution process, where each site maintains the same frequency profiles throughout the length of the tree. However, this simplifying assumption neglects the reality that site compositional shifts over time are an important aspect of sequence evolution, which may hinder accurate phylogenetic reconstruction (Galtier and Gouy, 1995). This clearly has special relevance in the identification of preference shifts using differences in stationary equilibrium between taxa, as discontinuities in the amino acid composition along the lineages are assumed to be the mechanic by which preference shifts occur.

Blanquart and Lartillot (2006) modelled compositional shift as break points, discrete events in which the profile vector $\pi$ of a site would shift along the tree. At a break point $\beta, \pi$ would switch to a new $\pi_{\beta}$ drawn from a uniform distribution, and then remain stationary until the next break point. A break point could be therefore be characterized in a standard substitution model by incorporating parameters $\pi_{\beta}$, the character of the break point change, $b_{\beta}$, the branch on which the break point occurs, and $x_{\beta}$, the point along the branch that it appears. The $Q_{\beta}$ rate matrix could then be calculated for each segment of the tree. Both the number and the position of break points were assumed to be Poisson-distributed along the tree. Blanquart and Lartillot (2008) later extended this model to a Bayesian MCMC context, incorporating the CAT model of Lartillot and Philippe (2004) for inference of $\pi_{\beta}$, thereby incorporating both site-heterogeneity and non-stationarity in a model denoted CATBP. All of this could be adapted to a mutation-selection context, as the underlying mathematical principles have already been laid out.

Though an infinite mixture model with a Dirichlet process prior (Lartillot and 
Philippe, 2004) lends itself well to directly inferring the substantially larger set of profiles contained in a BP model, this could also be adapted to a finite mixture model by using a single site-specific parameter that influenced the modulation of the allocated profile along the branch of the tree.

\subsection{Substitution Mapping}

Alternate approaches to detecting preference shifts can incorporate the use of substitution mappings, which characterize features in a lineage by identifying the substitution events that led to those features along the branches of the phylogenetic tree. Nielsen (2002) described a method of inferring substitution mappings by simulating ancestral states for each node in a tree, using the fractional likelihood of each nucleotide at that node based on the leaf states (Felsenstein, 1981). After generating these ancestral states, a substitution history through each node is sampled based on the ancestral state of the node and the state of its descendants. Nielsen (2002) uses a rejection-sampling method: if the final event along the substittuion history is not the same as the final node, the simulation is scrapped and started over.

Huelsenbeck et al. (2003) implemented this mapping approach in an MCMC context using stochastic sampling to generate the mutational paths, with an accept-reject algorithm based on whether the sampled node states in the reconstructed phylogeny matched the actual leaf node states. This was effective but extremely time-intensive, as the majority of ancestral states and mutational paths were rejected because they did not lead to valid leaf nodes.

Rodrigue et al. (2008) developed a method of generating substitution mappings which overcame these drawbacks as part of a data augmentation process in the PhyloBayes software (Lartillot et al. 2013). This method is based on a uniformization procedure described in Fearnhead and Sherlock (2006) and allows for a directed sim- 
ulation of substitution history. In this method the final state drawn along the substitution history is constrained to match the descendent node. Though this method may appear more computationally intensive, in some contexts such as codon models, it vastly outperforms Nielsen (2002)'s rejection scheme. The application to preference shift detection is clear, as it is these mapped substitution events that lead to variation in amino acid preferences between taxa.

\subsubsection{Preliminary Results}

As a contrast to finite mixture model approaches, we explored using substitution mappings to identify preference shifts. We generated mappings based on each sampler iteration in a 2000 cycle analysis of influenza PB2 using an amino-acid level GTR $+\Gamma$ model, which gave 2000 substitution mappings for each of the 759 coding sites. In each of these mappings, the branches of the trees were annotated with the nature and timing of each substitution event that led from the root state to each leaf node, allowing us to determine the number of substitutions that were inferred for each site (an analogue of the replacement rate for that site), and the time that the sampler spent in each amino acid state (analogous to the equilibrium distribution).

We used the mean proportion of time that the sampler spent in each amino acid state across all 2000 cycles to derive a proportional "frequency vector" of amino acids for each site. We then compared the Euclidean distance of these vectors between avian and human clades. Sites with no preference shift between clades would be expected to have similar proportions and therefore smaller distances, while sites with a shift would have larger distances. As these vectors were analogous to those in an empirical study of the same gene by Finkelstein et al. (2007), we used his criterion of a normalized Euclidean distance of 0.8 as marking a preference shift. We then applied this method to the same simulated datasets at various "peakedness" levels that we used to test our our MutSelBC profiles. The results are shown in figure 
3.1. At 0.999 peakedness, $98.9 \%$ of embedded shifts were identified, and $98.0 \%$ of sites without a shift were correctly identified. Comparison to MutSelBC models is

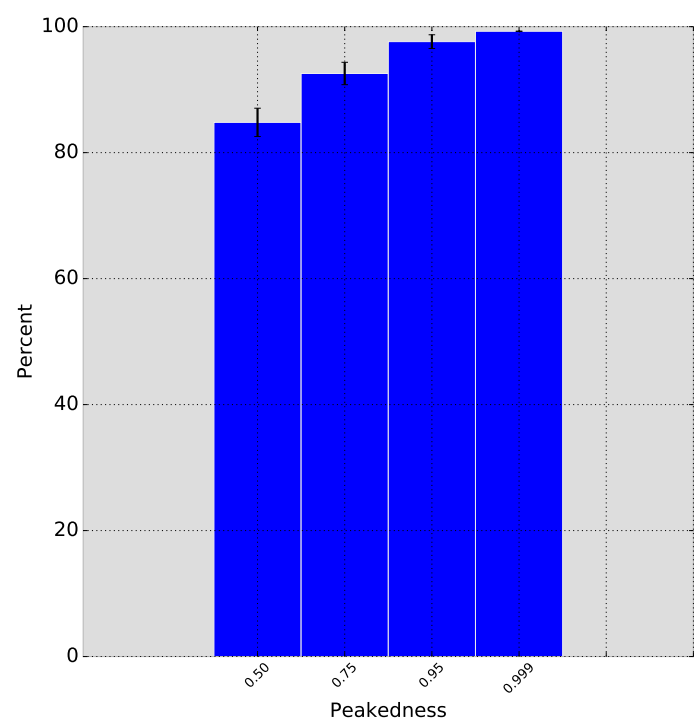

(a)

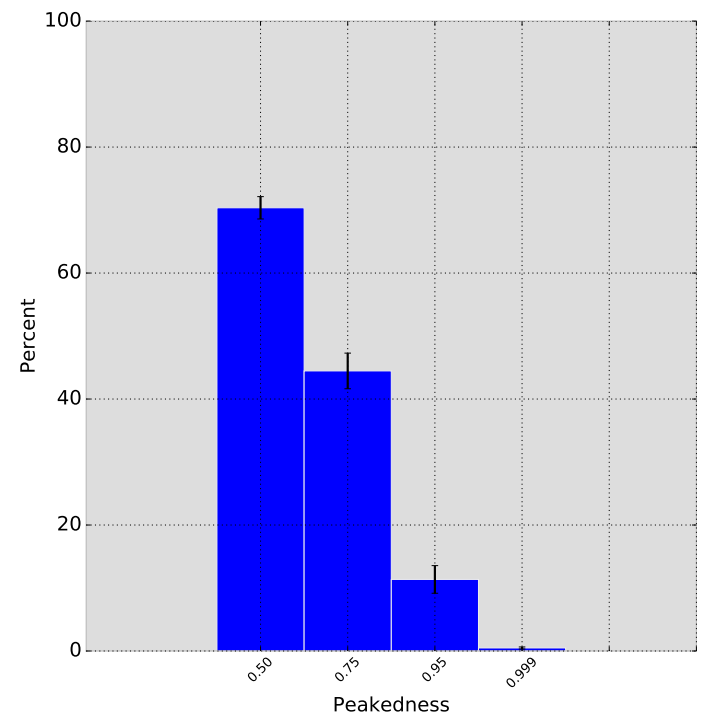

(c)

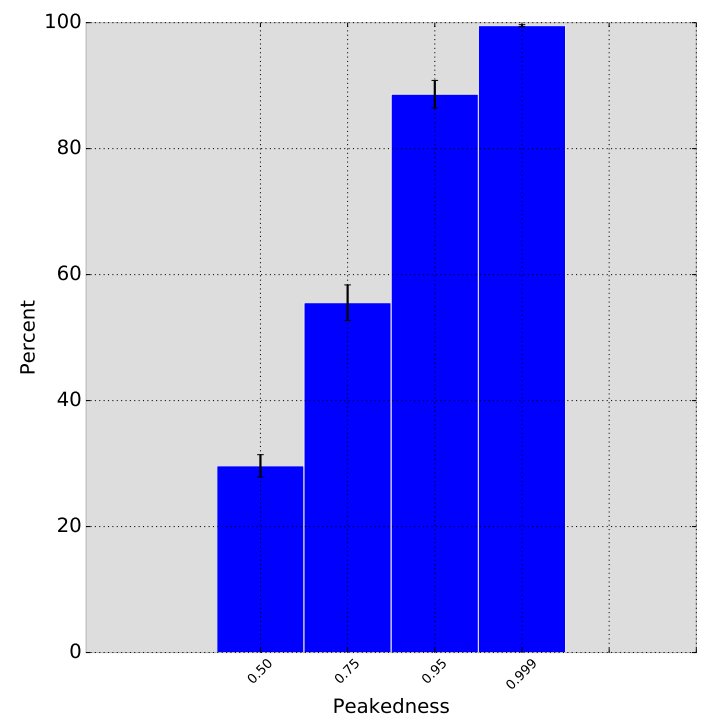

(b)

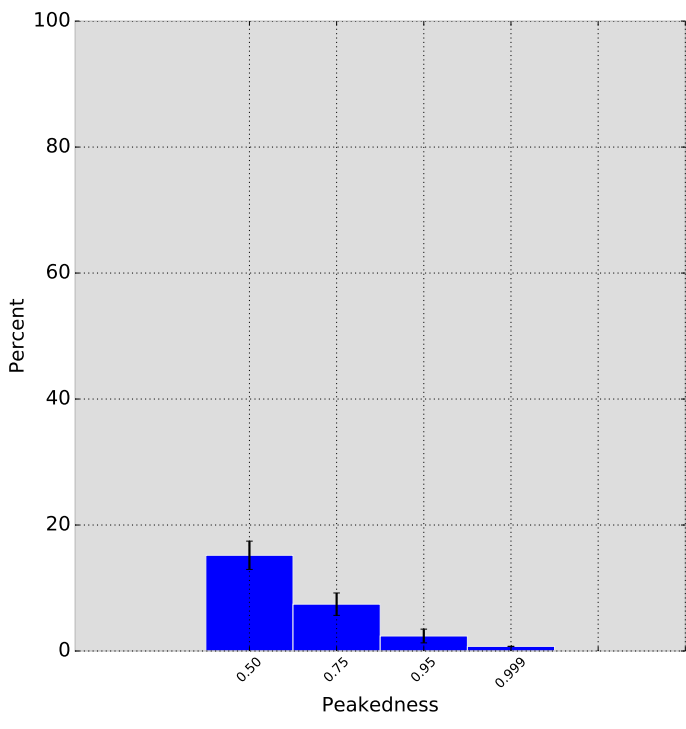

(d)

Figure 3.1: (a) True positive, (b) true negative, (c) false positive and (d) false negative detection of preference shifts for simulated data using substitution mapping.

interesting: at low peakedness, the substitution mapping method is much better at detecting true positives and avoiding false negatives than MutSelBC, while MutSelBC is better at detecting true negatives and avoiding false positives. It would appear that 
substitution mapping is a more sensitive but less specific when sites are under weak constraints or have higher variability.

A further useful feature of substitution mapping is in its freedom in selection of clades for comparison. Under the finite mixture model approach, each clade must be determined a priori and a separate MCMC analysis must be run on each clade's sequence alignment, in order to determine and compare site-specific profile allocation. In contrast, once substitution maps are generated for a phylogeny, taxa can be grouped and compared at will, as the branch-specific substitution history can be extracted for each sub-tree. This would allow data-driven investigation into site-specific differences for any feature present in the phylogeny, even differences that are not obvious above the protein-coding level. This could work in tandem with the mixture model analysis described in this paper, with substitution maps providing novel clade groupings that could then be analyzed by a complementary mixture model approach, to elaborate and confirm the site-specific preference shifts.

Preference shift identification as a indicator of constraint shifts is subject to founder effects, where a single shift in a progenitor can induce a permanent shift in a lineage, even if this is not biologically relevant to the protein's environment. This founder effect hypothesis could be tested via deep mutational scanning, a site-directed mutagenesis approach which correlates individual point mutations with organism viability (Doud et al. 2015; Bloom, 2017).

In conclusion, we have described multiple approaches to identifying clade-specific amino acid preference shifts, using finite mixture models in a Bayesian MCMC context. We have used established profile mixtures as well as novel, biochemically based profile mixtures. These mixture model approaches were contrasted with investigations on the same data by Tamuri et al. (2009) and Finkelstein et al. (2007), as well as our own amino-acid-level substitution mapping methods in an MCMC context, with favourable results. Future studies in this vein could include refinement of the 
mixture models used, incorporation of more complex substitution models, and expansion beyond the influenza avian-human host shift context to other viral and bacterial host shifts or indeed, any environmental shifts reflected by changes in amino acid preference at the molecular level. 


\section{Bibliography}

Baigent, Susan J, and John W McCauley. 2003. Influenza type a in humans, mammals and birds: Determinants of virus virulence, host-range and interspecies transmission. Bioessays 25:657-671.

Bazykin, Georgii A. 2015. Changing preferences: deformation of single position amino acid fitness landscapes and evolution of proteins. Biology letters 11:20150315.

Blanquart, Samuel, and Nicolas Lartillot. 2006. A bayesian compound stochastic process for modeling nonstationary and nonhomogeneous sequence evolution. Molecular Biology and Evolution 23:2058-2071.

Blanquart, Samuel, and Nicolas Lartillot. 2008. A site-and time-heterogeneous model of amino acid replacement. Molecular biology and evolution 25:842-858.

Bloom, Jesse D. 2017. Identification of positive selection in genes is greatly improved by using experimentally informed site-specific models. Biology direct 12:1.

Bruno, William J. 1996. Modeling residue usage in aligned protein sequences via maximum likelihood. Molecular Biology and Evolution 13:1368-1374.

Chen, Guang-Wu, Shih-Cheng Chang, Chee-Keng Mok, Yu-Luan Lo, Yu-Nong Kung, Ji-Hung Huang, Yun-Han Shih, Ji-Yi Wang, Chiayn Chiang, Chi-Jene Chen, et al. 2006. Genomic signatures of human versus avian influenza a viruses. Emerging infectious diseases 12:1353. 
Dos Reis, Mario, Philip CJ Donoghue, and Ziheng Yang. 2016. Bayesian molecular clock dating of species divergences in the genomics era. Nature Reviews. Genetics $17: 71$.

Dos Reis, Mario, Asif U Tamuri, Alan J Hay, and Richard A Goldstein. 2010. Charting the host adaptation of influenza viruses. Molecular biology and evolution 28:17551767.

Doud, Michael B, Orr Ashenberg, and Jesse D Bloom. 2015. Site-specific amino-acid preferences are mostly conserved in two closely related protein homologs. Molecular biology and evolution msv167.

Fearnhead, Paul, and Chris Sherlock. 2006. An exact gibbs sampler for the markovmodulated poisson process. Journal of the Royal Statistical Society: Series B (Statistical Methodology) 68:767-784.

Felsenstein, Joseph. 1981. Evolutionary trees from dna sequences: a maximum likelihood approach. Journal of molecular evolution 17:368-376.

Felsenstein, Joseph. 2001. Taking variation of evolutionary rates between sites into account in inferring phylogenies. Journal of Molecular Evolution 53:447-455.

Felsenstein, Joseph. 2004. Inferring phylogenies, volume 2. Sinauer associates Sunderland, MA.

Finkelstein, David B, Suraj Mukatira, Perdeep K Mehta, John C Obenauer, Xiaoping Su, Robert G Webster, and Clayton W Naeve. 2007. Persistent host markers in pandemic and h5n1 influenza viruses. Journal of virology 81:10292-10299.

Fitch, Walter M, and Emanuel Margoliash. 1967. A method for estimating the number of invariant amino acid coding positions in a gene using cytochrome $\mathrm{c}$ as a model case. Biochemical genetics 1:65-71. 
Galtier, Nicolas, and Manolo Gouy. 1995. Inferring phylogenies from dna sequences of unequal base compositions. Proceedings of the National Academy of Sciences 92:11317-11321.

Goldman, Nick, and Ziheng Yang. 1994. A codon-based model of nucleotide substitution for protein-coding dna sequences. Molecular biology and evolution 11:725-736.

Halpern, Aaron L, and William J Bruno. 1998. Evolutionary distances for proteincoding sequences: modeling site-specific residue frequencies. Molecular biology and evolution 15:910-917.

Hasegawa, Masami, Hirohisa Kishino, and Taka-aki Yano. 1985. Dating of the humanape splitting by a molecular clock of mitochondrial dna. Journal of molecular evolution 22:160-174.

Hastings, W Keith. 1970. Monte carlo sampling methods using markov chains and their applications. Biometrika 57:97-109.

Huelsenbeck, John P, Rasmus Nielsen, and Jonathan P Bollback. 2003. Stochastic mapping of morphological characters. Systematic Biology 52:131-158.

Jukes, Thomas H, Charles R Cantor, HN Munro, et al. 1969. Evolution of protein molecules. Mammalian protein metabolism 3:132.

Kimura, Motoo. 1980. A simple method for estimating evolutionary rates of base substitutions through comparative studies of nucleotide sequences. Journal of molecular evolution 16:111-120.

Lartillot, Nicolas, and Hervé Philippe. 2004. A bayesian mixture model for acrosssite heterogeneities in the amino-acid replacement process. Molecular biology and evolution 21:1095-1109. 
Lartillot, Nicolas, Nicolas Rodrigue, Daniel Stubbs, and Jacques Richer. 2013. Phylobayes mpi. phylogenetic reconstruction with infinite mixtures of profiles in a parallel environment. Systematic biology syt022.

Le, Si Quang, and Olivier Gascuel. 2008. An improved general amino acid replacement matrix. Molecular biology and evolution 25:1307-1320.

Metropolis, Nicholas, Arianna W Rosenbluth, Marshall N Rosenbluth, Augusta H Teller, and Edward Teller. 1953. Equation of state calculations by fast computing machines. The journal of chemical physics 21:1087-1092.

Miotto, Olivo, AT Heiny, Tin Wee Tan, J Thomas August, and Vladimir Brusic. 2008. Identification of human-to-human transmissibility factors in pb2 proteins of influenza a by large-scale mutual information analysis. BMC bioinformatics 9:S18.

Muse, Spencer V, and Brandon S Gaut. 1994. A likelihood approach for comparing synonymous and nonsynonymous nucleotide substitution rates, with application to the chloroplast genome. Molecular biology and evolution 11:715-724.

NCBI, Resource Coordinators. 2017. Database resources of the national center for biotechnology information. Nucleic acids research 45:D12.

Nielsen, Rasmus. 2002. Mapping mutations on phylogenies. Systematic biology $51: 729-739$.

Nilsson, Benjamin E, Aartjan JW te Velthuis, and Ervin Fodor. 2017. Role of the pb2 627 domain in influenza a virus polymerase function. Journal of Virology 91:e02467-16.

Parto, Sahar, and Nicolas Lartillot. 2017. Detecting consistent patterns of directional adaptation using differential selection codon models. BMC evolutionary biology 17:147. 
Quang, Le Si, Olivier Gascuel, and Nicolas Lartillot. 2008. Empirical profile mixture models for phylogenetic reconstruction. Bioinformatics 24:2317-2323.

Robert, Christian, and George Casella. 2010. Introducing monte carlo methods with $r$. Springer Science \& Business Media.

Rodrigue, N, and N Lartillot. 2017. Detecting adaptation in protein-coding genes using a bayesian site-heterogeneous mutation-selection codon substitution model. Molecular biology and evolution 34:204-214.

Rodrigue, Nicolas. 2013. On the statistical interpretation of site-specific variables in phylogeny-based substitution models. Genetics 193:557-564.

Rodrigue, Nicolas, and Stéphane Aris-Brosou. 2011. Fast bayesian choice of phylogenetic models: Prospecting data augmentation-based thermodynamic integration. Systematic biology 60:881-887.

Rodrigue, Nicolas, and Nicolas Lartillot. 2014. Site-heterogeneous mutation-selection models within the phylobayes-mpi package. Bioinformatics 30:1020-1021.

Rodrigue, Nicolas, Hervé Philippe, and Nicolas Lartillot. 2007. Exploring fast computational strategies for probabilistic phylogenetic analysis. Systematic biology $56: 711-726$.

Rodrigue, Nicolas, Hervé Philippe, and Nicolas Lartillot. 2008. Uniformization for sampling realizations of markov processes: applications to bayesian implementations of codon substitution models. Bioinformatics 24:56-62.

Rodrigue, Nicolas, Hervé Philippe, and Nicolas Lartillot. 2010. Mutation-selection models of coding sequence evolution with site-heterogeneous amino acid fitness profiles. Proceedings of the National Academy of Sciences 107:4629-4634. 
Roure, Béatrice, and Hervé Philippe. 2011. Site-specific time heterogeneity of the substitution process and its impact on phylogenetic inference. BMC evolutionary biology 11:1.

Tamuri, Asif U, Nick Goldman, and Mario dos Reis. 2014. A penalized-likelihood method to estimate the distribution of selection coefficients from phylogenetic data. Genetics 197:257-271.

Tamuri, Asif U, Mario dos Reis, and Richard A Goldstein. 2012. Estimating the distribution of selection coefficients from phylogenetic data using sitewise mutationselection models. Genetics 190:1101-1115.

Tamuri, Asif U, Mario dos Reis, Alan J Hay, and Richard A Goldstein. 2009. Identifying changes in selective constraints: host shifts in influenza. PLoS Comput Biol 5:e1000564.

Taubenberger, Jeffery K, Ann H Reid, Raina M Lourens, Ruixue Wang, et al. 2005. Characterization of the 1918 influenza virus polymerase genes. Nature 437:889.

Tavaré, Simon. 1986. Some probabilistic and statistical problems in the analysis of dna sequences. Lectures on mathematics in the life sciences 17:57-86.

Webster, Robert G, William J Bean, Owen T Gorman, Thomas M Chambers, and Yoshihiro Kawaoka. 1992. Evolution and ecology of influenza a viruses. Microbiological reviews 56:152-179.

Yang, Ziheng. 1993. Maximum-likelihood estimation of phylogeny from dna sequences when substitution rates differ over sites. Molecular biology and evolution 10:13961401.

Yang, Ziheng. 1994. Maximum likelihood phylogenetic estimation from dna sequences 
with variable rates over sites: approximate methods. Journal of Molecular evolution 39:306-314.

Yang, Ziheng. 1996. Among-site rate variation and its impact on phylogenetic analyses. Trends in Ecology $\&$ Evolution 11:367-372.

Yang, Ziheng. 1997. Paml: a program package for phylogenetic analysis by maximum likelihood. Bioinformatics 13:555-556.

Yang, Ziheng. 2014. Molecular evolution: a statistical approach. Oxford University Press.

Yang, Ziheng, and Rasmus Nielsen. 2008. Mutation-selection models of codon substitution and their use to estimate selective strengths on codon usage. Molecular biology and evolution 25:568-579. 